\title{
Diversity and Ecology of Diatoms in Pliocene Deposits of the Tunka Valley (Baikal Rift Zone)
}

\author{
Lyubov' Titova $^{1, *,+(\mathbb{D}) \text {, Abdulmonem Hassan }}{ }^{2,3,+}{ }^{\mathbb{D}}$, Ivan Mikhailov ${ }^{1}$, Elena Rodionova ${ }^{1}$, Sergei Rasskazov ${ }^{2,4}$ \\ and Marina Usoltseva ${ }^{1,+}$ (iD
}

1 Limnological Institute of the Siberian Branch of the Russian Academy of Sciences, 3 Ulan-Batorskaya, 664033 Irkutsk, Russia; mikhailov-89@mail.ru (I.M.); rodionova.73rodionova@yandex.ru (E.R.); usmarina@inbox.ru (M.U.)

2 Institute of the Earth's Crust of the Siberian Branch of the Russian Academy of Sciences, 128 Lermontova, 664033 Irkutsk, Russia; abdulmonemhassan86@gmail.com (A.H.); rassk@crust.irk.ru (S.R.)

3 Geology Department, Faculty of Science, Al-Baath University, Homs PB 77, Syria

4 Geological Faculty, Irkutsk State University, 3 Lenin, 664003 Irkutsk, Russia

* Correspondence: titova_873@mail.ru

+ These authors have an equal contribution to the study.

Citation: Titova, L.; Hassan, A.;

Mikhailov, I.; Rodionova, E.;

Rasskazov, S.; Usoltseva, M. Diversity and Ecology of Diatoms in Pliocene Deposits of the Tunka Valley (Baikal Rift Zone). Diversity 2021, 13, 479. https://doi.org/10.3390/d13100479

Academic Editor: Eduardo

Antonio Morales

Received: 5 August 2021

Accepted: 26 September 2021

Published: 29 September 2021

Publisher's Note: MDPI stays neutral with regard to jurisdictional claims in published maps and institutional affiliations.

Copyright: (c) 2021 by the authors. Licensee MDPI, Basel, Switzerland. This article is an open access article distributed under the terms and conditions of the Creative Commons Attribution (CC BY) license (https:// creativecommons.org/licenses/by/ $4.0 /)$.

\begin{abstract}
Fossil diatoms are an excellent tool for reconstructing the palaeoenvironmental and palaeogeographic changes involving lacustrine systems. In this work, the diatom content of Pliocene sediments recovered from a core extracted in the Tunka Basin (Baikal Rift Zone, Russia) is described. Revealed by light and scanning electron microscopy, 170 species of diatoms were found. Benthic, alkaliphilic, indifferent, cosmopolitan, and oligosaprobe species predominated. Ecological, geographical, and stratigraphic analysis of diatoms showed two ecozones, differing in taxonomic diversity of species. From the data obtained, palaeoenvironmental conditions of these zone formations have been reconstructed. It was shown that during the period corresponding to sedimentation in Ecozone II, the reservoir was cooler, as suggested by the increase of arctic-alpine taxa. The absence of Baikal Pliocene endemics and the presence of local endemics in the Tunka core indicate that there was no geographical connection between the palaeolake of the Tunka Valley and Lake Baikal during the Pliocene.
\end{abstract}

Keywords: diatom ecozone; Tunka Valley; Baikal; Pliocene; ecogeographical characteristics

\section{Introduction}

Lake Baikal is the deepest and oldest lacustrine basin in the world. Its waters fill an intermountain basin in the central part of the Baikal Rift System that has developed since the Oligocene [1,2]. Changes in the reservoir over such a long period can be traced through studying the palaeochronicle of diatoms. Global work on the study of the diatom palaeochronicle was carried out within the framework of the "Baikal Drilling" project [2]. The project was aimed at researching global changes in the natural environment and climate in Central Asia. It was shown that during the long history of the lake, the composition of the dominant complex of diatoms was repeatedly changed. Questions arise-what kind of flora developed in Lake Baikal and how was it distributed? The authors [2] suggested that diatom species penetrated into Lake Baikal through river input, mainly from the Transbaikalia and Tunka Valley basins.

The Tunka Valley is located in the southwestern part of the Baikal Rift Zone and consists of the following basins (from west to east): Mondy, Khoytogol, Turan, Tunka, Tory, and Bystraya, which are separated from each other by interbasin uplifts (spurs). The basins are filled with sediments of different origins. The most complete $2.1 \mathrm{~km}$ section, recorded by well P-2, shows mainly basalt and sandy pebble layers. Fine-grained (possibly lacustrine) sedimentary lenses, occurring in the lower part of the section, have coal interlayers. For 
palaeolimnological reconstructions, it is important to analyse compositions and spatialtemporal variations of diatom flora of the Tunka Basin (Figure 1), which occupies a central position in the valley and shows the deepest subsidence of the basement (up to $2500 \mathrm{~m}$ below sea level) [3].

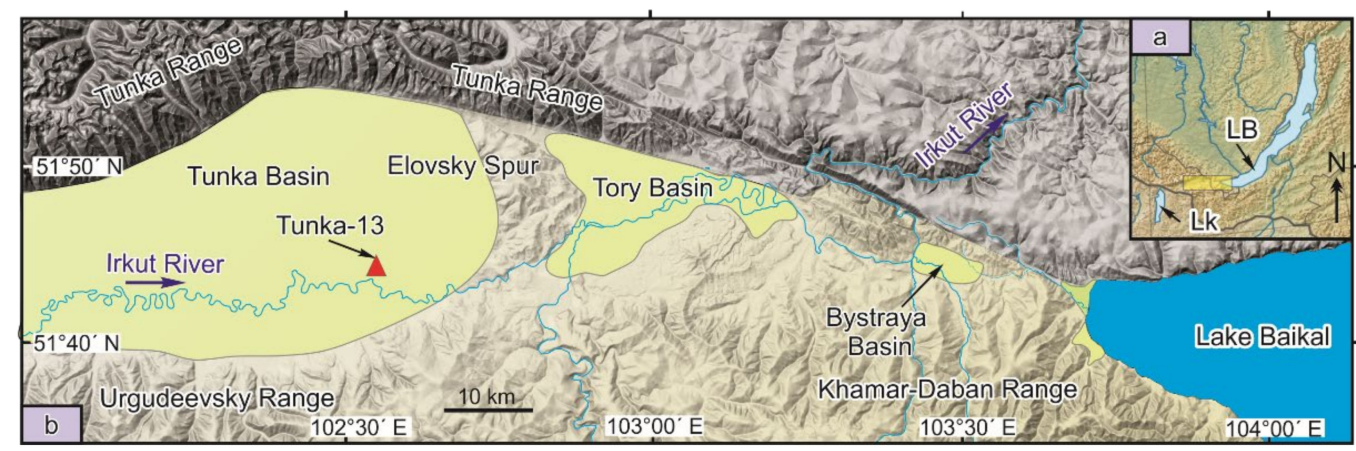

Figure 1. Stretching of the Tunka Rift Valley between the lacustrine Baikal (LB) and Khubsugul (LK) basins (a) and the location of the Tunka-13 drill hole in the central part of the rift valley within its largest Tunka Basin (b).

The eastern part of the valley that stretched from the Elovsky Spur to Lake Baikal experienced a change from extension to compression, which resulted in inversion from descending to ascending tectonic motions. Under sufficient extension, when the valley bottom was low, water of Lake Baikal could penetrate to its eastern or central parts $[4,5]$ (Figure 2).

\section{Tunka Basin Elovsky Spur Tory Basin Bystraya Basin Baikal Basin}

a

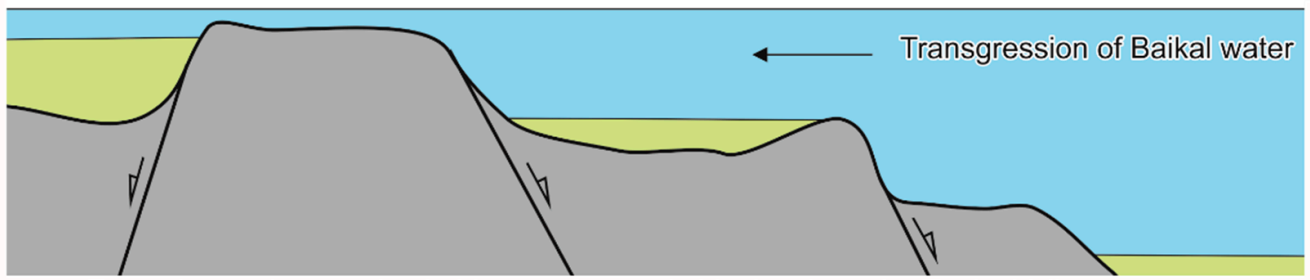

Extension and general subsidence of tectonic blocks along the Tunka Valley

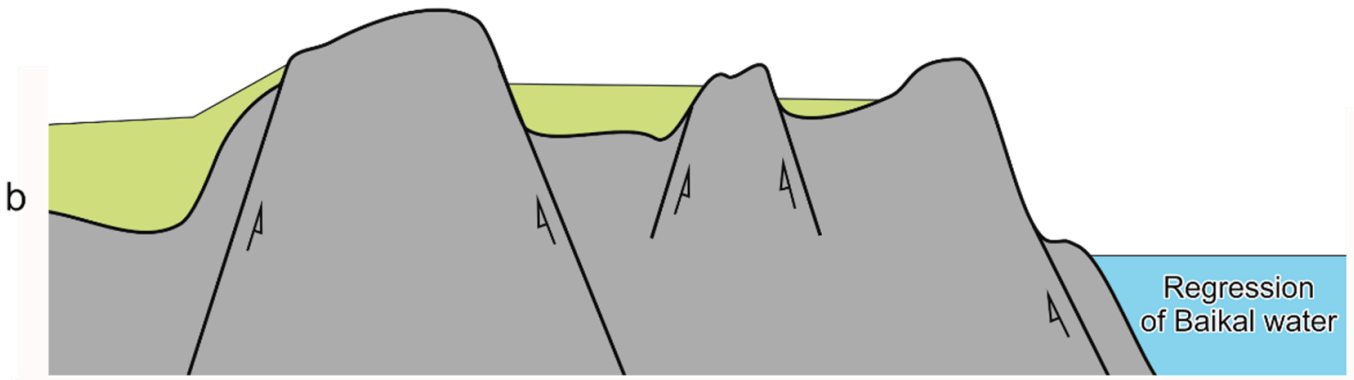

Compression and differentiated uplift of tectonic blocks along the Tunka Valley

Figure 2. Possible tectonic scenario of transgression (a) and regression (b) of water from Lake Baikal into the Tunka Valley. Extension of the crust along the Tunka Valley results in a general subsidence of tectonic blocks by normal faults in the Miocene-Early Pliocene. Subsequent compression entails an inversion of tectonic motions with a differentiated uplift of tectonic blocks by reverse faults.

Previously, complexes of Miocene and Pliocene diatom species from sediments of the Tunka Valley were described using light microscopy [6,7]. Subsequent work performed using scanning electron microscopy (SEM) allowed the authors to clarify the species composition and identify some new taxa [8-10]. 
Earlier, in order to obtain new data on the sedimentation of diatoms in the palaeoreservoir of the Tunka Valley, we drilled the Tunka-13 well and studied the composition of planktonic diatoms [11]. Benthic diatoms have not been studied by SEM. Since they are good indicators of ecological changes in the palaeowater body, their study is important for the Baikal region. At present, there are fragmentary data on the ecology of some fossil benthic diatoms of the Vitim Plateau in Transbaikalia [12], and there are no data on their species diversity in the Pliocene deposits of Lake Baikal.

Having studied the taxonomic composition of the Pliocene deposits of Baikal and Tunka, we can assume whether there was a connection between these reservoirs in the Pliocene.

In this work, we present new data on taxonomic diversity, stratigraphic distribution, and palaeoecological and palaeogeographic significance of fossil diatoms from sediments exposed by the Tunka-13 well in the Tunka basin.

\section{Materials and Methods}

\subsection{Core Sampling and Lithology}

Basins of the central part of the Baikal Rift Zone share a sedimentary succession of the Miocene-Lower Pliocene Tankhoi Formation, the Upper Pliocene-Eopleistocene ocherous Anosovka Formation, and the overlying tuffaceous-sedimentary and sandy units [13].

The Tunka-13 hole was drilled in the southeastern part of the Tunka Basin near Nikolskoye village (GPS coordinates: $51^{\circ} 43^{\prime} 45^{\prime \prime} \mathrm{N} ; 102^{\circ} 34^{\prime} 35^{\prime \prime} \mathrm{E}$ ). At the base of the section, an eroded lava flow is present that corresponds in composition to the horizon of basaltic lavas with $\mathrm{K}-\mathrm{Ar}$ ages of 16-15 Ma [11]. The overlying sediments reach an overall thickness of $86.5 \mathrm{~m}$.

In the section (Figure 3), nine units were identified from lithogeochemical data of sediments [11]. These units are attributable to the Tankhoi Formation (units 1-7) in the interval of 86.5-7.2 m, to the Anosovka Formation (unit 8) in the interval of 7.2-2.5 m, and to the lacustrine-aeolian sandy unit (unit 9) in the interval of the uppermost $2.5 \mathrm{~m}$. Between units 2 and 3 (in the depth interval of 66.1-71.0 m), a strongly watered loose sandy layer was found, which has not been studied.

The seven lower sedimentary members of the Tankhoi Formation show that the change of alluvial facies (units 1-3) through the avandelta diatom-free facies of a palaeolake (unit 4) to lacustrine facies is presented by grey and light grey aleurolite enriched in fossil diatoms (units 5-7). The eighth unit (interval $2.7-6.6 \mathrm{~m}$ ) designates the alluvial facies of the Anosovka Formation, the ninth one (interval $<2.4 \mathrm{~m}$ ), the accumulation of the final lacustrine-aeolian sandy sediments [11].

These sediments have accumulated since the Miocene-Pliocene boundary on the western slope of the uplifted Elovsky interbasin spur. The accumulated Middle-Upper Miocene lava sequence was eroded almost to the bottom, and the erosion slot was filled with alluvial deposits generated due to erosion of basaltic material at the end of the Late Miocene [11].

From palynological data, we earlier referred the oldest sediments of the section to the end of the Late Miocene- the beginning of the Pliocene [11]. 


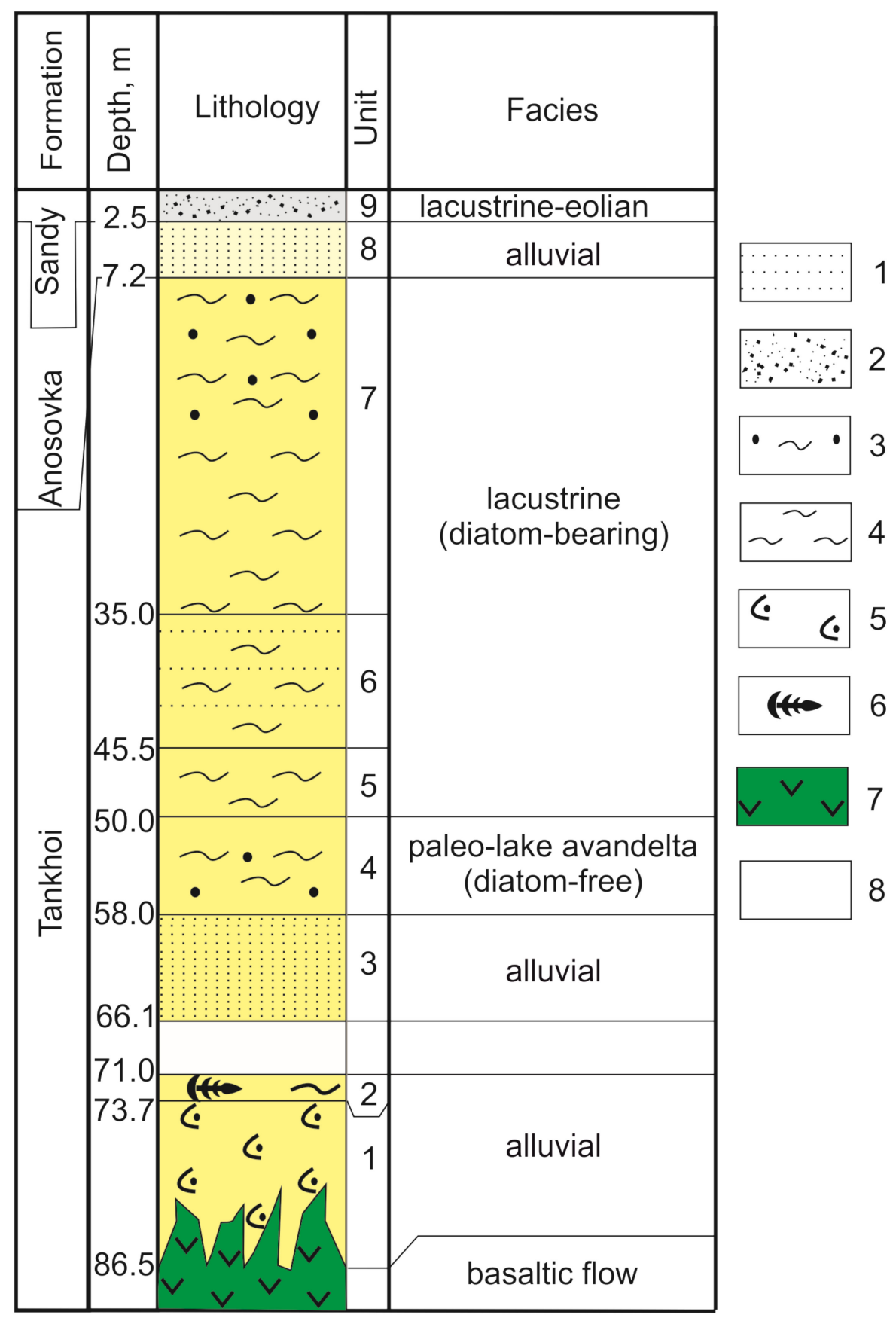

Figure 3. Stratigraphic columns of the Tunka-13 drill hole. 1-sandstone; 2-sand; 3-aleuritic sandstone; 4-aleurolite; 5-basaltic tuffites; 6-phytodetritus; 7—basalt; 8-no core.

\subsection{Diatom Analysis}

For diatom analysis, 63 sedimentary deposit samples were taken from the core. Samples were prepared for light microscopy and a quantitative account was performed according to the method described in [14]. Cleaned valves were dried on cover slips and mounted in Naphrax (Naphrax Ltd., Bedford, UK, refractive index $=1.74$ ) and all the diatom valves found on the slides were counted using light microscopy Axiovert 200 ZEISS LM (Carl Zeiss, Jena, Germany) equipped with a Pixera Penguin 600CL camera.

For SEM observations, sediment samples were cleaned in $30 \% \mathrm{H}_{2} \mathrm{O}_{2}$ solution at $75^{\circ} \mathrm{C}$ for $3 \mathrm{~h}$, rinsed three times with deionised water, then centrifuged and rinsed several times in $0.1 \%$ sodium diphosphate anhydrous with distilled water to remove clay particles. Cleaned slurry was then mounted on a brass stub and coated with gold using an SDC 004 
(BALZERS) ion sputter for $150 \mathrm{~s}$ at 10-15 mA. The stub was analysed using an SEM Quanta 200 (FEI Company, Hillsboro, OR, USA) at $21.5 \mathrm{kV}$ and $10 \mathrm{~mm}$ working distance.

All SEM pictures were mounted using Adobe Photoshop CS4 Portable (Adobe Inc., San Jose, CA, USA). The Venn diagram was constructed using a free tool at resource http:/ / bioinformatics.psb.ugent.be/webtools/Venn/ (accessed on 21 July 2021).

Morphological identification of taxa was carried out using the literature [2,10,15-35]. All determinations were performed while taking into account recent taxonomic changes listed in AlgaeBase [36]. Ecological-geographic analyses were performed according to the articles [37-45].

\section{Results}

\subsection{Diatom Flora}

The taxonomic diversity of the identified diatom flora was relatively high; in total, 170 diatom taxa (Table 1) were identified. They relate to 57 genera, 27 families, 15 orders, and 3 classes of the phylum Bacillariophyta. The diatom flora consisted of $96.5 \%$ recent and $3.5 \%$ extinct species.

A detailed taxonomic list of the diatom flora is reported in Table 1. The dominant families are the following: Achnanthidiaceae (17 species), Staurosiraceae (14), Cocconeidaceae (13), Naviculaceae (13), Eunotiaceae (12), Pinnulariaceae (12), Gomphonemataceae (11), Fragilariaceae (10), Tabellariaceae (10), Aulacoseiraceae (6), Sellaphoraceae (6), Bacillariaceae (5), Catenulaceae (5), Cavinulaceae (5), Ulnariaceae (5), Stauroneidaceae (4), Neidiaceae (4), Diploneidaceae (3), and Rhopalodiaceae (3). Other families are represented by 1-2 species. The dominant genera are Eunotia Ehrenberg (12), Pinnularia Ehrenberg (12), Navicula Bory (9), Staurosira Ehrenberg (8), Planothidium Round \& L. Bukhtiyarova (8), Gomphonema Ehrenberg (6), Aulacoseira Thwaites (6), Amphora Ehrenberg ex Kützing (5), Fragilaria Lyngbye (5), Cavinula D.G. Mann \& A.J. Stickle (5), and Cymbella C. Agardh (5). 
Table 1. List of diatom taxa with ecological characteristics.

\begin{tabular}{|c|c|c|c|c|c|c|c|c|c|c|c|c|c|}
\hline \multirow{2}{*}{ № } & \multirow{2}{*}{ Taxon } & \multicolumn{3}{|c|}{$\begin{array}{l}\text { Diatom } \\
\text { Ecozone }\end{array}$} & \multicolumn{8}{|c|}{ Ecological and Geographical Characteristics of Indicator Organisms } & \multirow{2}{*}{$\begin{array}{l}\text { Fossil \& } \\
\text { Recent }\end{array}$} \\
\hline & & I & II & Hab & $\mathrm{T}$ & $\mathbf{R}$ & S1 & S2 & Sal & $\mathrm{pH}$ & A & Geo & \\
\hline \multicolumn{14}{|c|}{$\begin{array}{l}\text { Phylum Bacillariophyta } \\
\text { Class Bacillariophyceae } \\
\text { Subclass Bacillariophycidae } \\
\text { Order Bacillariales } \\
\text { Family Bacillariaceae }\end{array}$} \\
\hline 2. & Nitzschia alpina Hustedt & - & + & B & - & - & sx & $\mathrm{o}$ & - & - & acf & $\mathrm{b}$ & $\mathrm{R}$ \\
\hline 3. & N. fonticola (Grunow) Grunow & - & + & $\mathrm{B}$ & - & - & - & $0-\beta$ & $\mathrm{i}$ & 7.7 & alf & $\mathrm{k}$ & $\mathrm{R}$ \\
\hline 4. & N. frustulum (Kützing) Grunow & - & + & $\mathrm{B}$ & - & - & - & - & $\mathrm{hl}$ & - & - & $\mathrm{b}$ & $\mathrm{R}$ \\
\hline 5. & N. recta Hantzsch ex Rabenhorst & - & + & $\mathrm{B}$ & & st & es & $\mathrm{x}$ & $\mathrm{i}$ & $6-9$ & alf & $\mathrm{k}$ & $\mathrm{R}$ \\
\hline \multicolumn{14}{|c|}{$\begin{array}{l}\text { Order Cocconeidales } \\
\text { Family Achnanthidiaceae }\end{array}$} \\
\hline 7. & A. minutissimum (Kützing) Czarnecki & + & + & $\mathrm{B}$ & eterm & st-str & es & $\beta$ & $\mathrm{i}$ & $4.3-9.2$ & alf & $\mathrm{k}$ & $\mathrm{R}$ \\
\hline 8. & A. obliquum Mereschkowsky & + & + & $\mathrm{B}$ & - & - & - & - & - & - & - & - & $\mathrm{R}$ \\
\hline 9. & Eucocconeis flexella (Kützing) Meister & - & + & $\mathrm{B}$ & - & - & sx & o & $\mathrm{mh}$ & - & ind & $\mathrm{a}-\mathrm{a}$ & $\mathrm{R}$ \\
\hline 10. & E. laevis (Østrup) Lange-Bertalot & + & - & $\mathrm{B}$ & - & - & - & o & - & - & - & - & $\mathrm{R}$ \\
\hline 11. & $\begin{array}{l}\text { Planothidium dubium (Grunow) Round \& } \\
\text { Bukhtiyarova }\end{array}$ & - & + & $\mathrm{B}$ & - & - & sx & - & $\mathrm{i}$ & - & alf & - & $\mathrm{R}$ \\
\hline 12. & P. ellipticum (Cleve) M.B. Edlund & + & - & $\mathrm{B}$ & - & str & sx & - & $\mathrm{i}$ & - & alf & $\mathrm{k}$ & $\mathrm{R}$ \\
\hline 13. & $\begin{array}{l}\text { P. frequentissimum (Lange-Bertalot) } \\
\text { Lange-Bertalot }\end{array}$ & - & + & $\mathrm{B}$ & - & - & - & $\mathrm{x}-\mathrm{O}$ & oh & - & alf & $\mathrm{k}$ & $\mathrm{R}$ \\
\hline 14. & P. haynaldii (Schaarschmidt) Lange-Bertalot & + & - & $\mathrm{B}$ & - & - & sx & $\beta-\alpha$ & - & - & alf & $\mathrm{k}$ & $\mathrm{R}$ \\
\hline 15. & P. joursacense (Héribaud) Lange-Bertalot & + & + & $\mathrm{B}$ & - & - & sx & - & - & - & - & - & $\mathrm{R}$ \\
\hline 16. & $\begin{array}{l}\text { P. lanceolatum (Brébisson ex Kützing) } \\
\text { Lange-Bertalot }\end{array}$ & + & + & P-B & warm & st-str & sx & $x-0$ & $\mathrm{i}$ & $7.5-8.1$ & alf & $\mathrm{k}$ & $\mathrm{R}$ \\
\hline
\end{tabular}


Table 1. Cont.

\begin{tabular}{|c|c|c|c|c|c|c|c|c|c|c|c|c|c|}
\hline \multirow[t]{2}{*}{ № } & \multirow{2}{*}{ Taxon } & \multicolumn{3}{|c|}{$\begin{array}{l}\text { Diatom } \\
\text { Ecozone }\end{array}$} & \multicolumn{8}{|c|}{ Ecological and Geographical Characteristics of Indicator Organisms } & \multirow{2}{*}{$\begin{array}{l}\text { Fossil \& } \\
\text { Recent }\end{array}$} \\
\hline & & I & II & Hab & $\mathrm{T}$ & $\mathbf{R}$ & S1 & S2 & Sal & $\mathrm{pH}$ & $\mathbf{A}$ & Geo & \\
\hline 17. & P. linkei (Hustedt) Lange-Bertalot & + & + & B & - & - & - & - & $\mathrm{hl}$ & - & - & - & $\mathrm{R}$ \\
\hline 18. & P. rostratum (Østrup) Lange-Bertalot & - & + & B & - & - & sx & - & - & - & - & - & $\mathrm{R}$ \\
\hline 19. & $\begin{array}{l}\text { Psammothidium marginulatum (Grunow) } \\
\text { Bukhtiyarova \& Round }\end{array}$ & - & + & $\mathrm{B}$ & - & st-str & sx & $\beta-\mathrm{o}$ & - & 5.4 & acf & $\mathrm{a}, \mathrm{k}$ & $\mathrm{R}$ \\
\hline 21. & $\begin{array}{l}\text { Skabitschewskia oestrupii (A. Cleve) Kulikovskiy \& } \\
\text { Lange-Bertalot }\end{array}$ & - & + & B & - & - & - & $\mathrm{o}$ & $\mathrm{i}$ & - & ind & $\mathrm{a}-\mathrm{a}$ & $\mathrm{R}$ \\
\hline 22. & $\begin{array}{l}\text { S. peragalloi (Brun \& Héribaud) Kulikovskiy \& } \\
\text { Lange-Bertalot }\end{array}$ & - & + & B & - & - & sx & $\mathrm{o}$ & $\mathrm{i}$ & - & ind & $\mathrm{b}$ & $\mathrm{R}$ \\
\hline \multicolumn{14}{|c|}{ Family Cocconeidaceae } \\
\hline 23. & Cocconeis placentula Ehrenberg & + & + & P-B & temp & st-str & es & $0-\beta$ & $\mathrm{i}$ & $5.5-9$ & alf & $\mathrm{k}$ & $\mathrm{R}$ \\
\hline 25. & Cymbella aff. laevis Nägeli & + & - & B & cool & - & sx & - & $\mathrm{i}$ & - & ind & $\mathrm{b}$ & $\mathrm{R}$ \\
\hline 26. & C. amplificata Krammer & - & + & $\mathrm{B}$ & - & - & - & - & $\mathrm{i}$ & - & - & $\mathrm{b}$ & $\mathrm{R}$ \\
\hline 27. & C. cistula (Ehrenberg) O. Kirchner & - & + & $\mathrm{B}$ & - & st-str & sx & $\mathrm{o}-\beta$ & $\mathrm{i}$ & 8 & alf & $\mathrm{k}$ & $\mathrm{R}$ \\
\hline 28. & C. cymbiformis C. Agardh & - & + & $\mathrm{B}$ & temp & - & sx & - & $\mathrm{i}$ & $6.2-9$ & alf & $\mathrm{k}$ & $\mathrm{R}$ \\
\hline 29. & C. stuxbergii (Cleve) Cleve & - & + & $\mathrm{B}$ & - & - & - & - & - & - & - & - & $\mathrm{R}$ \\
\hline 30. & Cymbopleura cuspidata (Kützing) Krammer & + & + & B & temp & - & - & $0-\alpha$ & $\mathrm{i}$ & 6.7 & ind & $\mathrm{k}$ & $\mathrm{R}$ \\
\hline 31. & C. incerta (Grunow) Krammer & - & + & B & - & - & - & - & $\mathrm{i}$ & - & ind & $\mathrm{a}-\mathrm{a}$ & $\mathrm{R}$ \\
\hline 32. & C. subcuspidata (Krammer) Krammer & - & + & $\mathrm{B}$ & - & - & - & $\mathrm{o}$ & $\mathrm{i}$ & - & ind & $\mathrm{a}-\mathrm{a}$ & $\mathrm{R}$ \\
\hline 33. & Didymosphenia geminata (Lyngbye) Mart. Schmidt & - & + & $\mathrm{B}$ & - & st-str & sx & $\mathrm{x}$ & $\mathrm{i}$ & - & ind & $\mathrm{a}-\mathrm{a}$ & $\mathrm{R}$ \\
\hline 34. & Gomphonella olivacea (Hornemann) Rabenhorst & + & + & $\mathrm{B}$ & - & - & es & $\beta-\alpha$ & $\mathrm{i}$ & $7.5-8$ & alf & $\mathrm{k}$ & $\mathrm{R}$ \\
\hline
\end{tabular}


Table 1. Cont.

\begin{tabular}{|c|c|c|c|c|c|c|c|c|c|c|c|c|c|}
\hline \multirow{2}{*}{ № } & \multirow{2}{*}{ Taxon } & \multicolumn{3}{|c|}{$\begin{array}{l}\text { Diatom } \\
\text { Ecozone }\end{array}$} & \multicolumn{8}{|c|}{ Ecological and Geographical Characteristics of Indicator Organisms } & \multirow{2}{*}{$\begin{array}{l}\text { Fossil \& } \\
\text { Recent }\end{array}$} \\
\hline & & $\mathbf{I}$ & II & Hab & $\mathrm{T}$ & $\mathbf{R}$ & S1 & S2 & Sal & $\mathrm{pH}$ & A & Geo & \\
\hline \multicolumn{14}{|c|}{$\begin{array}{l}\text { Order Cymbellales } \\
\text { Family Anomoeoneidaceae }\end{array}$} \\
\hline 35. & $\begin{array}{l}\text { Adlafia minuscula var. muralis (Grunow) } \\
\text { Lange-Bertalot }\end{array}$ & - & + & $\mathrm{B}$ & - & - & $\mathrm{sp}$ & $0-\beta$ & $\mathrm{i}$ & - & ind & $\mathrm{k}$ & $\mathrm{R}$ \\
\hline \multicolumn{14}{|c|}{ Family Gomphonemataceae } \\
\hline 36. & Encyonema minutum (Hilse) D.G. Mann & - & + & $\mathrm{B}$ & - & st-str & es & $0-\beta$ & oh & 6.2 & ind & $\mathrm{k}$ & $\mathrm{R}$ \\
\hline 37. & E. silesiacum (Bleisch) D.G. Mann & - & + & B & - & st-str & sx & $\mathrm{x}-\mathrm{o}$ & $\mathrm{i}$ & $6.2-7.7$ & ind & $\mathrm{k}$ & $\mathrm{R}$ \\
\hline 38. & Encyonopsis cesatii (Rabenhorst) Krammer & - & + & B & - & - & - & $\mathrm{x}-\mathrm{O}$ & - & - & - & - & $\mathrm{R}$ \\
\hline 39. & Gomphonema angustatum (Kützing) Rabenhorst & + & + & P-B & - & - & - & $\beta$ & $\mathrm{i}$ & - & alf & $\mathrm{k}$ & $\mathrm{R}$ \\
\hline 40. & G. intricatum Kützing & + & + & P-B & - & - & - & $\mathrm{x}-\mathrm{O}$ & - & - & ind & $\mathrm{k}$ & $\mathrm{R}$ \\
\hline 41. & G. acuminatum Ehrenberg & - & + & P-B & & - & - & $x-\beta$ & $\mathrm{i}$ & - & alf & $\mathrm{k}$ & $\mathrm{R}$ \\
\hline 42. & G. olivaceum var. minutissimum Hustedt & + & + & $\mathrm{B}$ & - & - & - & - & $\mathrm{i}$ & - & alf & $\mathrm{b}$ & $\mathrm{R}$ \\
\hline 43. & G. sphaerophorum Ehrenberg & + & + & $\mathrm{B}$ & - & - & - & $\mathrm{o}$ & $\mathrm{i}$ & - & alf & $\mathrm{b}$ & $\mathrm{R}$ \\
\hline 44. & G. ventricosum W. Gregory & - & + & $\mathrm{P}$ & cool & - & - & $\mathrm{o}-\mathrm{x}$ & $\mathrm{i}$ & - & ind & $\mathrm{k}$ & $\mathrm{R}$ \\
\hline 45. & Placoneis exigua (W. Gregory) Mereschkovsky & - & + & B & - & - & es & $\mathrm{x}-\mathrm{O}$ & $\mathrm{i}$ & - & alf & $\mathrm{k}$ & $\mathrm{R}$ \\
\hline 46. & P. gastrum (Ehrenberg) Mereschkowsky & + & + & $\mathrm{B}$ & - & - & sx & $\mathrm{x}-\mathrm{O}$ & $\mathrm{i}$ & - & ind & $\mathrm{k}$ & $\mathrm{R}$ \\
\hline 47. & Reimeria sinuata (W. Gregory) Kociolek \& Stoermer & - & + & $\mathrm{B}$ & - & st & sx & - & $\mathrm{i}$ & - & ind & $\mathrm{k}$ & $\mathrm{R}$ \\
\hline \multicolumn{14}{|c|}{ Family Rhoicospheniaceae } \\
\hline 48. & $\begin{array}{l}\text { Gomphosphenia grovei var. lingulata (Hustedt) } \\
\text { Lange-Bertalot }\end{array}$ & + & + & B & - & str & es & $\beta-\alpha$ & $\mathrm{i}$ & - & - & $\mathrm{k}$ & $\mathrm{F}$ \\
\hline \multicolumn{14}{|c|}{$\begin{array}{l}\text { Order Eunotiales } \\
\text { Family Eunotiaceae }\end{array}$} \\
\hline 49. & $\begin{array}{l}\text { Eunotia aff. exigua (Brébisson ex Kützing) } \\
\text { Rabenhorst }\end{array}$ & - & + & B & - & - & es & $o-\beta$ & $\mathrm{hb}$ & $3.4-8$ & acf & $\mathrm{k}$ & $\mathrm{R}$ \\
\hline
\end{tabular}


Table 1. Cont.

\begin{tabular}{|c|c|c|c|c|c|c|c|c|c|c|c|c|c|}
\hline \multirow{2}{*}{ № } & \multirow{2}{*}{ Taxon } & \multicolumn{3}{|c|}{$\begin{array}{l}\text { Diatom } \\
\text { Ecozone }\end{array}$} & \multicolumn{8}{|c|}{ Ecological and Geographical Characteristics of Indicator Organisms } & \multirow{2}{*}{$\begin{array}{l}\text { Fossil \& } \\
\text { Recent }\end{array}$} \\
\hline & & $\mathbf{I}$ & II & Hab & $\mathrm{T}$ & $\mathbf{R}$ & S1 & S2 & Sal & $\mathrm{pH}$ & A & Geo & \\
\hline 50. & E. aff. spatulata J. Veselá\& J.R. Johansen & - & + & $\mathrm{B}$ & - & - & - & - & - & - & - & - & $\mathrm{R}$ \\
\hline 51. & E. arcus Ehrenberg & - & + & $\mathrm{B}$ & - & - & - & $x-\beta$ & $\mathrm{i}$ & - & acf & $\mathrm{k}$ & $\mathrm{R}$ \\
\hline 52. & E. bidens Ehrenberg & + & - & B & cool & - & - & - & $\mathrm{hb}$ & - & acf & $\mathrm{k}$ & $\mathrm{R}$ \\
\hline 53. & E. incisa W. Smith ex W. Gregory & - & + & $\mathrm{B}$ & - & str & es & $\alpha-\beta$ & - & 5.0 & acf & $\mathrm{k}$ & $\mathrm{R}$ \\
\hline 55. & E. pectinalis (Kützing) Rabenhorst & - & + & B & temp & - & - & $\begin{array}{l}x, x-0 \\
x-\beta\end{array}$ & $\mathrm{hb}$ & $5.8-7.0$ & ind & $\mathrm{k}$ & $\mathrm{R}$ \\
\hline 56. & E. polydentula Hustedt & - & + & B & - & - & - & $x-\beta$ & $\mathrm{hb}$ & - & acf & $\mathrm{k}$ & $\mathrm{R}$ \\
\hline 57. & E. praerupta Ehrenberg & - & + & P-B & cool & st-str & sx & $\beta$ & - & - & acf & $\mathrm{k}$ & $\mathrm{R}$ \\
\hline 58. & E. robusta Ralfs & + & + & B & - & - & - & $\mathrm{o}$ & - & - & - & - & $\mathrm{R}$ \\
\hline 59. & E. tenella (Grunow) Hustedt & + & + & B & - & str & es & $0-\beta$ & $\mathrm{hb}$ & 5.1 & acf & $\mathrm{a}-\mathrm{a}$ & $\mathrm{R}$ \\
\hline \multicolumn{14}{|c|}{$\begin{array}{l}\text { Order Fragilariales } \\
\text { Family Fragilariaceae }\end{array}$} \\
\hline 61. & $\begin{array}{l}\text { Fragilaria aff. pararumpens Lange-Bertalot, } \\
\text { G. Hofmann \& Werum }\end{array}$ & - & + & $\mathrm{P}-\mathrm{B}$ & - & - & - & - & - & - & - & - & $\mathrm{R}$ \\
\hline 62. & F. capucina Desmazières & + & + & $\mathrm{B}$ & - & - & es & o & $\mathrm{i}$ & 7.7 & alf & $\mathrm{k}$ & $\mathrm{R}$ \\
\hline 63. & F. radians (Kützing) D.M. Williams \& Round & - & + & B & - & st-str & sx & - & - & - & - & $\mathrm{Ha}, \mathrm{Pt}$ & $\mathrm{R}$ \\
\hline 64. & F. aequalis Heiberg & - & + & $\mathrm{B}$ & - & - & - & - & - & - & - & - & $\mathrm{R}$ \\
\hline 65. & F. vaucheriae (Kützing) J.B. Petersen & - & + & P-B & - & - & sx & $\mathrm{o}-\beta$ & $\mathrm{i}$ & 7.8 & alf & $\mathrm{k}$ & $\mathrm{R}$ \\
\hline 66. & $\begin{array}{l}\text { Fragilariforma constricta (Ehrenberg) } \\
\text { D.M. Williams \& Round }\end{array}$ & - & + & $\mathrm{B}$ & - & - & - & - & $\mathrm{i}$ & 5.2 & acf & $\mathrm{a}-\mathrm{a}$ & $\mathrm{R}$ \\
\hline 67. & F. virescens (Ralfs) D.M. Williams \& Round & - & + & P-B & - & st & es & o & i & 6.8 & ind & $\mathrm{k}$ & $\mathrm{R}$ \\
\hline 68. & Odontidium hyemale (Roth) Kützing & - & + & $\mathrm{P}-\mathrm{B}$ & cool & st-str & sx & $\beta-o$ & $\mathrm{hb}$ & $6.5-7.5$ & ind & $\mathrm{k}$ & $\mathrm{R}$ \\
\hline
\end{tabular}


Table 1. Cont.

\begin{tabular}{|c|c|c|c|c|c|c|c|c|c|c|c|c|c|}
\hline \multirow[t]{2}{*}{ № } & \multirow{2}{*}{ Taxon } & \multicolumn{3}{|c|}{$\begin{array}{l}\text { Diatom } \\
\text { Ecozone }\end{array}$} & \multicolumn{8}{|c|}{ Ecological and Geographical Characteristics of Indicator Organisms } & \multirow{2}{*}{$\begin{array}{l}\text { Fossil \& } \\
\text { Recent }\end{array}$} \\
\hline & & I & II & Hab & $\mathrm{T}$ & $\mathbf{R}$ & S1 & S2 & Sal & $\mathrm{pH}$ & $\mathbf{A}$ & Geo & \\
\hline 69. & O. mesodon (Kützing) Kützing & + & + & B & cool & st-str & sx & $0-\beta$ & $\mathrm{hb}$ & - & - & $\mathrm{k}$ & $\mathrm{R}$ \\
\hline 70. & $\begin{array}{l}\text { Punctastriata lancettula (Schumann) } \\
\text { P.B. Hamilton \& Siver }\end{array}$ & + & + & $\mathrm{B}$ & cool & - & es & $\mathrm{o}$ & $\mathrm{i}$ & 7.8 & alb & $\mathrm{b}$ & $\mathrm{R}$ \\
\hline \multicolumn{14}{|c|}{ Family Staurosiraceae } \\
\hline 72. & $\begin{array}{l}\text { P. elliptica (Schumann) Edlund, E. Morales \& } \\
\text { Spaulding }\end{array}$ & + & + & B & - & - & - & $\beta-\alpha$ & - & - & - & $\mathrm{k}$ & $\mathrm{R}$ \\
\hline 73. & P. parasitica (W. Smith) E. Morales & - & + & B & - & - & - & $\beta-\alpha$ & - & - & - & - & $\mathrm{R}$ \\
\hline 74. & $\begin{array}{l}\text { Staurosira aff. leptostauron (Ehrenberg) } \\
\text { Kulikovskiy \& Genkal }\end{array}$ & + & + & $\mathrm{B}$ & - & - & - & - & - & - & - & - & $\mathrm{R}$ \\
\hline 75. & S. venter (Ehrenberg) Cleve \& J.D. Möller & + & - & P-B & warm & st-str & sx & $\beta$ & $\mathrm{i}$ & $5.5-9$ & alf & $\mathrm{k}$ & $\mathrm{R}$ \\
\hline 77. & S. construens Ehrenberg & + & - & P-B & temp & st-str & sx & $\mathrm{o}$ & $\mathrm{i}$ & $5.5-9$ & alf & $\mathrm{k}$ & $\mathrm{R}$ \\
\hline 78. & $\begin{array}{l}\text { S. construens var. triundulata (Reichelt) } \\
\text { Bukhtiyarova }\end{array}$ & + & + & B & - & - & - & - & $\mathrm{i}$ & - & alf & $\mathrm{k}$ & $\mathrm{R}$ \\
\hline 79. & S. leptostauron (Ehrenberg) Kulikovskiy \& Genkal & + & + & $\mathrm{B}$ & - & st & es & $\alpha-\beta$ & $\mathrm{hb}$ & 8.4 & alf & $\mathrm{b}$ & $\mathrm{R}$ \\
\hline 80. & S. subsalina (Hustedt) Lange-Bertalot & - & + & P-B & - & st & es & $\mathrm{o}$ & $\mathrm{hl}$ & - & alf & $\mathrm{k}$ & $\mathrm{R}$ \\
\hline 81. & S. tabellaria (W. Smith) Leuduger-Fortmorel & + & - & $\mathrm{B}$ & - & - & - & - & - & - & - & - & $\mathrm{R}$ \\
\hline 82. & $\begin{array}{l}\text { Staurosirella lanceolata (Hustedt) E. Morales, } \\
\text { C.Wetzel \& L.Ector }\end{array}$ & + & + & B & - & - & - & - & - & - & - & - & $\mathrm{R}$ \\
\hline 83. & $\begin{array}{l}\text { S. martyi (Héribaud) E. Morales \& } \\
\text { K.M. Manoylov }\end{array}$ & + & + & P-B & - & st-str & es & $0-\alpha$ & $\mathrm{i}$ & $7.5-9$ & alf & $\mathrm{k}$ & $\mathrm{R}$ \\
\hline 84. & S. pinnata (Ehrenberg) D.M. Williams \& Round & + & + & B & temp & st-str & es & $\beta-\alpha$ & $\mathrm{hl}$ & $6.2-9.3$ & alf & $\mathrm{k}$ & $\mathrm{R}$ \\
\hline
\end{tabular}


Table 1. Cont.

\begin{tabular}{|c|c|c|c|c|c|c|c|c|c|c|c|c|c|}
\hline \multirow[t]{2}{*}{ № } & \multirow{2}{*}{ Taxon } & \multicolumn{3}{|c|}{$\begin{array}{l}\text { Diatom } \\
\text { Ecozone }\end{array}$} & \multicolumn{8}{|c|}{ Ecological and Geographical Characteristics of Indicator Organisms } & \multirow{2}{*}{$\begin{array}{l}\text { Fossil \& } \\
\text { Recent }\end{array}$} \\
\hline & & $\mathbf{I}$ & II & $\mathrm{Hab}$ & $\mathrm{T}$ & $\mathbf{R}$ & S1 & S2 & Sal & $\mathrm{pH}$ & A & Geo & \\
\hline \multicolumn{14}{|c|}{$\begin{array}{l}\text { Order Licmophorales } \\
\text { Family Ulnariaceae }\end{array}$} \\
\hline 85. & Hannaea arcus (Ehrenberg) R.M. Patrick & - & + & B & & str & es & o & $\mathrm{i}$ & - & alf & $\mathrm{a}-\mathrm{a}$ & $\mathrm{R}$ \\
\hline 86. & H. baicalensis Genkal, Popovskaya \& Kulikovskiy & - & + & $\mathrm{P}$ & - & - & - & - & - & - & - & - & $\mathrm{R}$ \\
\hline 88. & U. contracta (Østrup) E. Morales \& M.L. Vis & - & + & $\mathrm{P}$ & - & - & es & - & - & - & - & - & $\mathrm{R}$ \\
\hline 89. & U. ulna (Nitzsch) Compère & + & + & P-B & - & - & - & $0-\alpha$ & $\mathrm{i}$ & - & alf & $\mathrm{k}$ & $\mathrm{R}$ \\
\hline \multicolumn{14}{|c|}{$\begin{array}{l}\text { Order Naviculales } \\
\text { Family Amphipleuraceae }\end{array}$} \\
\hline 90. & Frustulia vulgaris (Thwaites) De Toni & - & + & P-B & - & st & es & $x-\beta$ & $\mathrm{i}$ & - & alf & $\mathrm{k}$ & $\mathrm{R}$ \\
\hline \multicolumn{14}{|c|}{ Family Brachysiraceae } \\
\hline 91. & $\begin{array}{l}\text { Nupela impexiformis (Lange-Bertalot) } \\
\text { Lange-Bertalot }\end{array}$ & - & + & $\mathrm{B}$ & - & - & es & - & - & 7.0 & ind & - & $\mathrm{R}$ \\
\hline \multicolumn{14}{|c|}{ Family Cavinulaceae } \\
\hline 92. & $\begin{array}{l}\text { Cavinula cocconeiformis (W.Gregory ex Greville) D.G. } \\
\text { Mann \& A.J. Stickle }\end{array}$ & + & + & P-B & - & str & es & o & $\mathrm{i}$ & 6.9 & ind & $\mathrm{a}-\mathrm{a}$ & $\mathrm{R}$ \\
\hline 93. & C. jaernefeltii (Hustedt) D.G. Mann \& A.J. Stickle & + & + & $\mathrm{B}$ & - & - & - & $\mathrm{o}$ & $\mathrm{i}$ & - & acf & $\mathrm{k}$ & $\mathrm{R}$ \\
\hline 94. & C. pseudoscutiformis (Hustedt) D.G. Mann \& Stickle & - & + & P-B & - & st-str & sx & o & $\mathrm{i}$ & 6.7 & ind & $\mathrm{a}-\mathrm{a}$ & $\mathrm{R}$ \\
\hline 95. & C. scutelloides (W. Smith) Lange-Bertalot & + & + & $\mathrm{B}$ & - & - & - & $0-\beta$ & - & - & - & - & $\mathrm{R}$ \\
\hline 96. & C. scutiformis (Grunow) D.G. Mann \& A.J. Stickle & + & + & $\mathrm{B}$ & - & - & - & - & $\mathrm{i}$ & - & ind & $\mathrm{a}-\mathrm{a}$ & $\mathrm{R}$ \\
\hline \multicolumn{14}{|c|}{ Family Diploneidaceae } \\
\hline 97. & Diploneis elliptica (Kützing) Cleve & + & - & B & temp & & sx & $\mathrm{o}-\mathrm{a}$ & $\mathrm{i}$ & - & alf & $\mathrm{k}$ & $\mathrm{R}$ \\
\hline 98. & D. ovalis (Hilse) Cleve & + & + & B & - & - & $\mathrm{sp}$ & $\beta$ & $\mathrm{i}$ & $6.5-9$ & alb & $\mathrm{b}$ & $\mathrm{R}$ \\
\hline 99. & D. parma Cleve & + & - & $\mathrm{B}$ & cool & - & - & $0-\beta$ & $\mathrm{i}$ & - & alf & $\mathrm{Ha}$ & $\mathrm{R}$ \\
\hline
\end{tabular}


Table 1. Cont.

\begin{tabular}{|c|c|c|c|c|c|c|c|c|c|c|c|c|c|}
\hline \multirow{2}{*}{ № } & \multirow{2}{*}{ Taxon } & \multicolumn{3}{|c|}{$\begin{array}{l}\text { Diatom } \\
\text { Ecozone }\end{array}$} & \multicolumn{8}{|c|}{ Ecological and Geographical Characteristics of Indicator Organisms } & \multirow{2}{*}{$\begin{array}{l}\text { Fossil \& } \\
\text { Recent }\end{array}$} \\
\hline & & $\mathbf{I}$ & II & $\mathrm{Hab}$ & $\mathrm{T}$ & $\mathbf{R}$ & S1 & S2 & Sal & $\mathrm{pH}$ & A & Geo & \\
\hline \multicolumn{14}{|c|}{ Family Naviculaceae } \\
\hline 100. & Caloneis bacillum (Grunow) Cleve & - & + & $\mathrm{B}$ & temp & - & es & o & $\mathrm{i}$ & - & alf & $\mathrm{k}$ & $\mathrm{R}$ \\
\hline 101. & Gyrosigma acuminatum (Kützing) Rabenhorst & - & + & $\mathrm{B}$ & cool & - & - & $0-x$ & $\mathrm{i}$ & - & alf & $\mathrm{k}$ & $\mathrm{R}$ \\
\hline 102. & $\begin{array}{l}\text { Hippodonta costulata (Grunow) Lange-Bertalot, } \\
\text { Metzeltin \& Witkowski }\end{array}$ & + & - & B & temp & - & sx & - & $\mathrm{hl}$ & - & alf & $\mathrm{b}$ & $\mathrm{R}$ \\
\hline 103. & H. coxiae Lange-Bertalot & - & + & $\mathrm{B}$ & - & - & - & - & - & - & alf & - & $\mathrm{R}$ \\
\hline 104. & Navicula cryptocephala Kützing & + & + & P-B & - & - & - & $\mathrm{x}$ & $\mathrm{i}$ & - & alf & $\mathrm{k}$ & $\mathrm{R}$ \\
\hline 105. & $\begin{array}{l}\text { N. cryptotenella Lange-Bertalot in Krammer \& } \\
\text { Lange-Bertalot }\end{array}$ & - & + & P-B & - & - & - & $\mathrm{b}-\mathrm{a}$ & $\mathrm{i}$ & - & alf & $\mathrm{k}$ & $\mathrm{R}$ \\
\hline 106. & N. gregaria Donkin & + & - & $\mathrm{B}$ & - & - & es & $x-\beta$ & $\mathrm{mh}$ & - & alf & $\mathrm{k}$ & $\mathrm{R}$ \\
\hline 107. & $\begin{array}{l}\text { N. johncarteri D.M. Williams in D.M. Williams \& G. } \\
\text { Reid }\end{array}$ & + & + & B & - & - & sx & $\mathrm{x}$ & - & - & - & - & $\mathrm{R}$ \\
\hline 109. & $\begin{array}{l}\text { N. meniscula var. muralis (Grunow) } \\
\text { Lange Bertalot }\end{array}$ & - & + & B & & & & & & & & & $\mathrm{R}$ \\
\hline 110. & N. radiosa Kützing & + & + & $\mathrm{B}$ & temp & st-str & es & $\mathrm{o}$ & $\mathrm{i}$ & $5 .-9$ & ind & $\mathrm{k}$ & $\mathrm{R}$ \\
\hline 111. & N. rhynchocephala Kützing & - & + & $\mathrm{B}$ & - & - & - & $\beta$ & $\mathrm{hl}$ & $7.3-7.8$ & alf & $\mathrm{k}$ & $\mathrm{R}$ \\
\hline 112. & N. viridula (Kützing) Ehrenberg & + & - & $\mathrm{B}$ & - & - & es & $\mathrm{o}$ & $\mathrm{hl}$ & - & alf & $\mathrm{k}$ & $\mathrm{R}$ \\
\hline \multicolumn{14}{|c|}{ Family Neidiaceae } \\
\hline 113. & Neidium baicalense Jasnitsky & - & + & $\mathrm{B}$ & - & - & - & - & $\mathrm{i}$ & - & ind & - & $\mathrm{R}$ \\
\hline 114. & N. bisulcatum (Lagerstedt) Cleve & - & + & B & - & - & es & $\mathrm{o}-\beta$ & $\mathrm{hb}$ & 5.2 & ind & $\mathrm{b}$ & $\mathrm{R}$ \\
\hline 115. & N. dubium (Ehenberg) Cleve & - & + & $\mathrm{B}$ & - & - & - & $\mathrm{x}$ & $\mathrm{i}$ & - & alf & $\mathrm{k}$ & $\mathrm{R}$ \\
\hline 116. & N. longiceps (W. Gregory) R. Ross & - & + & B & - & - & $\mathrm{sp}$ & $\mathrm{o}$ & $\mathrm{i}$ & 6.1 & acf & $\mathrm{a}-\mathrm{a}$ & $\mathrm{R}$ \\
\hline
\end{tabular}


Table 1. Cont.

\begin{tabular}{|c|c|c|c|c|c|c|c|c|c|c|c|c|c|}
\hline \multirow{2}{*}{ № } & \multirow{2}{*}{ Taxon } & \multicolumn{3}{|c|}{$\begin{array}{l}\text { Diatom } \\
\text { Ecozone }\end{array}$} & \multicolumn{8}{|c|}{ Ecological and Geographical Characteristics of Indicator Organisms } & \multirow{2}{*}{$\begin{array}{l}\text { Fossil \& } \\
\text { Recent }\end{array}$} \\
\hline & & $\mathbf{I}$ & II & $\mathrm{Hab}$ & $\mathrm{T}$ & $\mathbf{R}$ & S1 & S2 & Sal & $\mathrm{pH}$ & A & Geo & \\
\hline \multicolumn{14}{|c|}{ Family Sellaphoraceae } \\
\hline 117. & Eolimna aboensis (Cleve) S.I. Genkal & + & + & $\mathrm{B}$ & - & - & - & - & $\mathrm{i}$ & - & ind & $\mathrm{a}-\mathrm{a}$ & $\mathrm{R}$ \\
\hline 118. & E. minima (Grunow) Lange-Bertalot & + & + & $\mathrm{B}$ & - & - & - & $0-\beta$ & - & - & - & - & $\mathrm{R}$ \\
\hline 119. & Sellaphora bacillum (Ehrenberg) D.G. Mann & + & - & B & - & st-str & sx & $\mathrm{x}-\mathrm{O}$ & $\mathrm{i}$ & 7.-9 & alf & $\mathrm{k}$ & $\mathrm{R}$ \\
\hline 121. & S. pupula (Kützing) Mereschkovsky & - & + & $\mathrm{B}$ & eterm & st & $\mathrm{sp}$ & $0-x$ & hl & $5.2-9$ & ind & $\mathrm{k}$ & $\mathrm{R}$ \\
\hline 122. & $\begin{array}{l}\text { S. stauroneioides (Lange-Bertalot) Veselá \& } \\
\text { J.R.Johansen }\end{array}$ & + & - & B & - & - & - & - & - & - & - & - & $\mathrm{R}$ \\
\hline \multicolumn{14}{|c|}{ Family Stauroneidaceae } \\
\hline 123. & $\begin{array}{l}\text { Craticula subminuscula (Manguin) C.E. Wetzel \& } \\
\text { Ector }\end{array}$ & - & + & P-B & - & - & $\mathrm{sp}$ & $\alpha-\beta$ & - & - & - & - & $\mathrm{R}$ \\
\hline 124. & Stauroneis anceps Ehrenberg & + & + & P-B & - & - & - & $\mathrm{x}$ & $\mathrm{i}$ & - & ind & $\mathrm{k}$ & $\mathrm{R}$ \\
\hline 125. & S. phoenicenteron (Nitzsch) Ehrenberg & - & + & $\mathrm{B}$ & temp & - & es & $x-0$ & $\mathrm{i}$ & 7.3 & ind & $\mathrm{k}$ & $\mathrm{R}$ \\
\hline 126. & S. smithii Grunow & + & + & P-B & - & st-str & - & $x-0$ & - & - & alf & $\mathrm{k}$ & $\mathrm{R}$ \\
\hline \multicolumn{14}{|c|}{ Family Pinnulariaceae } \\
\hline 127. & $\begin{array}{l}\text { Pinnularia abaujensis var. linearis (Hustedt) R.M. } \\
\text { Patrick }\end{array}$ & + & - & B & - & - & - & - & $\mathrm{i}$ & - & ind & $\mathrm{b}$ & $\mathrm{R}$ \\
\hline 128. & P.aff. interrupta W. Smith & - & + & $\mathrm{B}$ & - & - & $\mathrm{sp}$ & $\beta-o$ & $\mathrm{i}$ & 5.6 & acf & $\mathrm{k}$ & $\mathrm{R}$ \\
\hline 129. & P. brebissonii (Kützing) Rabenhorst & + & - & B & - & - & es & $\mathrm{o}-\beta$ & - & - & - & $\mathrm{k}$ & $\mathrm{R}$ \\
\hline 130. & P. eifeliana (Krammer) Krammer & - & + & $\mathrm{B}$ & - & - & - & o & - & - & - & - & $\mathrm{R}$ \\
\hline 131. & P. interrupta var. minutissima Hustedt & - & + & B & - & - & - & - & $\mathrm{i}$ & - & - & $\mathrm{b}$ & $\mathrm{R}$ \\
\hline 132. & P. microstauron (Ehrenberg) Cleve & + & + & B & temp & - & $\mathrm{sp}$ & $\mathrm{x}$ & $\mathrm{i}$ & - & ind & $\mathrm{k}$ & $\mathrm{R}$ \\
\hline 133. & P. biundulata (O.Müller) Kulikovskiy \& Genkal & - & + & B & - & - & - & - & - & - & - & - & $\mathrm{R}$ \\
\hline 134. & P. nodosa (Ehrenberg) W. Smith & - & + & B & - & - & - & o & $\mathrm{i}$ & - & ind & $\mathrm{a}-\mathrm{a}$ & $\mathrm{R}$ \\
\hline
\end{tabular}


Table 1. Cont.

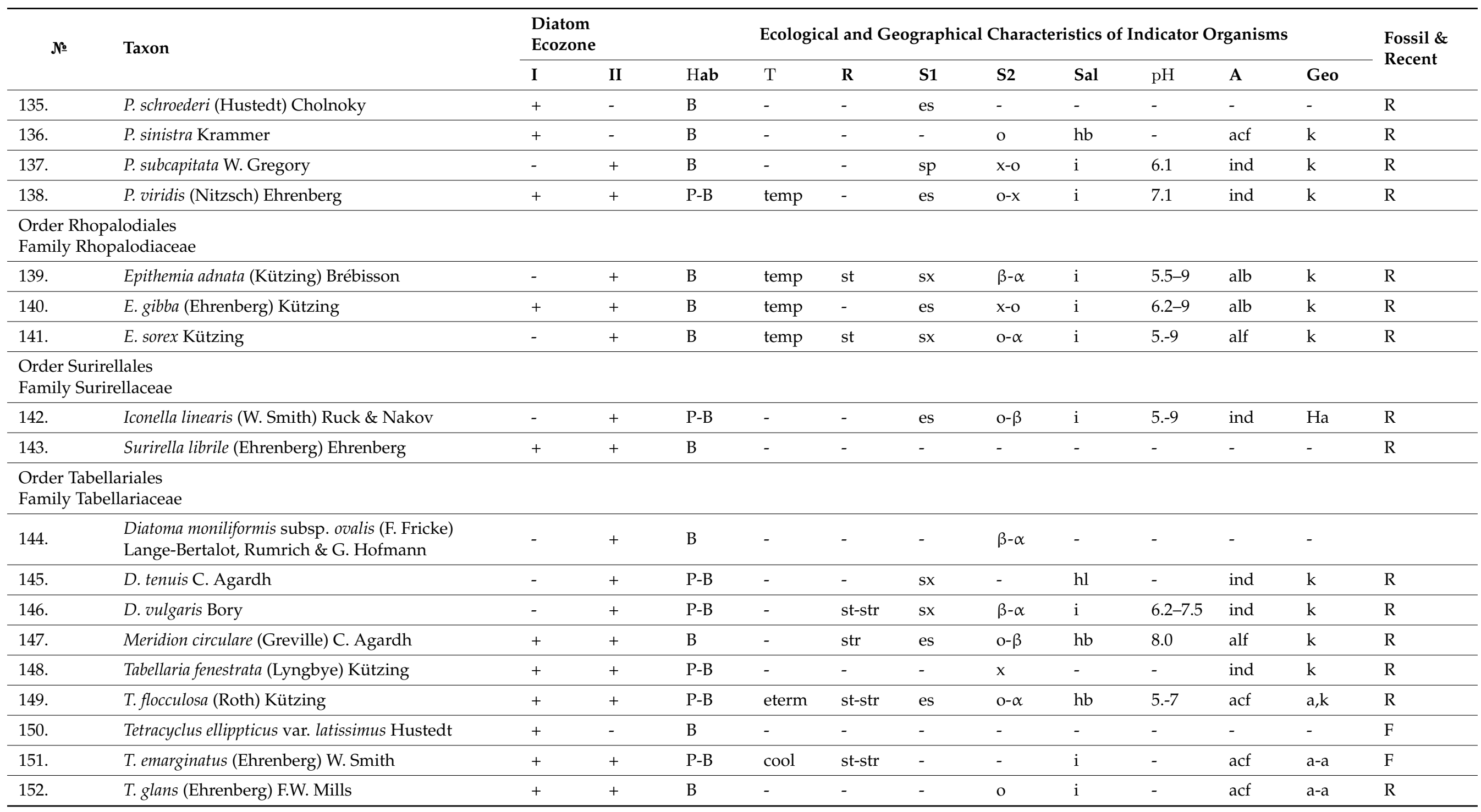


Table 1. Cont.

\begin{tabular}{|c|c|c|c|c|c|c|c|c|c|c|c|c|c|}
\hline \multirow[t]{2}{*}{ № } & \multirow{2}{*}{ Taxon } & \multicolumn{3}{|c|}{$\begin{array}{l}\text { Diatom } \\
\text { Ecozone }\end{array}$} & \multicolumn{8}{|c|}{ Ecological and Geographical Characteristics of Indicator Organisms } & \multirow{2}{*}{$\begin{array}{l}\text { Fossil \& } \\
\text { Recent }\end{array}$} \\
\hline & & I & II & Hab & $\mathrm{T}$ & $\mathbf{R}$ & S1 & S2 & Sal & $\mathrm{pH}$ & $\mathbf{A}$ & Geo & \\
\hline 153. & T. lapponicus Tynni & + & + & B & - & - & - & - & - & - & - & - & $\mathrm{F}$ \\
\hline \multicolumn{14}{|c|}{$\begin{array}{l}\text { Order Thalassiophysales } \\
\text { Family Catenulaceae }\end{array}$} \\
\hline 154. & Amphora aequalis Krammer & - & + & B & - & - & - & - & - & - & - & - & $\mathrm{R}$ \\
\hline 155. & A.aff. inariensis Krammer & + & - & B & - & - & - & $0-\beta$ & oh & - & alf & $\mathrm{Ha}$ & $\mathrm{R}$ \\
\hline 156. & A. lybica Ehrenberg & - & + & B & - & - & es & - & $\mathrm{hl}$ & - & alf & $\mathrm{k}$ & $\mathrm{R}$ \\
\hline 157. & A. ovalis (Kützing) Kützing & + & - & B & temp & st-str & sx & $\alpha-\beta$ & $\mathrm{i}$ & $6.2-9$ & alf & $\mathrm{k}$ & $\mathrm{R}$ \\
\hline 158. & A. pediculus (Kützing) Grunow & - & + & $\mathrm{B}$ & temp & st & es & $0-\alpha$ & $\mathrm{i}$ & - & alf & $\mathrm{k}$ & $\mathrm{R}$ \\
\hline \multicolumn{14}{|c|}{$\begin{array}{l}\text { Class Coscinodiscophyceae } \\
\text { Order Aulacoseirales } \\
\text { Family Aulacoseiraceae }\end{array}$} \\
\hline 159. & Aulacoseira aff. baicalensis (Wislouch) Simonsen & + & + & $\mathrm{P}$ & - & - & - & - & - & - & - & - & $\mathrm{R}$ \\
\hline 161. & A. ambigua f. curvata (Skabichevskij) Genkal & + & + & $\mathrm{P}$ & - & st-str & $\mathrm{sp}$ & $0-\beta$ & $\mathrm{i}$ & 7.1 & alb & $\mathrm{k}$ & $\mathrm{R}$ \\
\hline 162. & A. distans (Ehrenberg) Simonsen & + & + & P-B & $\mathrm{cool}$ & - & $\mathrm{sp}$ & $\mathrm{x}-\mathrm{O}$ & $\mathrm{i}$ & 6.9 & acf & $\mathrm{b}$ & $\mathrm{R}$ \\
\hline 163. & A. islandica (O. Müller) Simonsen & + & - & $\mathrm{P}$ & cool & - & es & $0-x$ & $\mathrm{i}$ & - & acf & $\mathrm{b}$ & $\mathrm{R}$ \\
\hline 164. & A. subarctica (O. Müller) E.Y. Haworth & + & - & $\mathrm{P}$ & - & st-str & - & $\alpha-\beta$ & $\mathrm{i}$ & 7.3 & alb & $\mathrm{a}, \mathrm{k}$ & $\mathrm{R}$ \\
\hline \multicolumn{14}{|c|}{$\begin{array}{l}\text { Order Melosirales } \\
\text { Family Melosiraceae }\end{array}$} \\
\hline 165. & Melosira undulata (Ehrenberg) Kützing & + & + & P-B & temp & - & - & $0-\alpha$ & $\mathrm{i}$ & - & alb & $\mathrm{k}$ & $\mathrm{R}$ \\
\hline \multicolumn{14}{|c|}{$\begin{array}{l}\text { Order Paraliales } \\
\text { Family Radialiplicataceae }\end{array}$} \\
\hline 166. & Ellerbeckia arenaria f. teres (Brun) R.M. Crawford & + & + & P-B & - & st-str & - & $0-\alpha$ & $\mathrm{i}$ & - & alf & $\mathrm{k}$ & $\mathrm{R}$ \\
\hline
\end{tabular}


Table 1. Cont.

\begin{tabular}{|c|c|c|c|c|c|c|c|c|c|c|c|c|c|}
\hline \multirow{2}{*}{ № } & \multirow{2}{*}{ Taxon } & \multicolumn{3}{|c|}{$\begin{array}{l}\text { Diatom } \\
\text { Ecozone }\end{array}$} & \multicolumn{8}{|c|}{ Ecological and Geographical Characteristics of Indicator Organisms } & \multirow{2}{*}{$\begin{array}{l}\text { Fossil \& } \\
\text { Recent }\end{array}$} \\
\hline & & I & II & Hab & $\mathrm{T}$ & $\mathbf{R}$ & S1 & S2 & Sal & $\mathrm{pH}$ & A & Geo & \\
\hline \multicolumn{14}{|c|}{$\begin{array}{l}\text { Class Mediophyceae } \\
\text { Order Stephanodiscales } \\
\text { Family Stephanodiscaceae }\end{array}$} \\
\hline 167. & $\begin{array}{l}\text { Cyclotella tuncaica Nikiteeeva, Likhoshway \& } \\
\text { Pomazkina }\end{array}$ & + & + & $\mathrm{P}$ & - & - & - & - & - & - & - & - & $\mathrm{F}$ \\
\hline 168. & $\begin{array}{l}\text { Stephanodiscus tuncaensis Pomazkina \& } \\
\text { Likhoshway }\end{array}$ & + & + & $\mathrm{P}$ & - & - & - & - & - & - & - & - & $\mathrm{F}$ \\
\hline \multicolumn{14}{|c|}{$\begin{array}{l}\text { Class Bacillariophyta classis incertae sedis } \\
\text { Order Bacillariophyta ordo incertae sedis } \\
\text { Family Bacillariophyta familia incertae sedis }\end{array}$} \\
\hline 169. & $\begin{array}{l}\text { Gliwiczia calcar (Cleve) M. Kulikovskiy, } \\
\text { Lange-Bertalot \& A. Witkowski }\end{array}$ & + & + & B & - & - & - & o & $\mathrm{i}$ & - & alf & $\mathrm{a}-\mathrm{a}$ & $\mathrm{R}$ \\
\hline 170. & Navigeia decussis (Østrup) Bukhtiyarova & + & - & B & - & - & es & $0-\alpha$ & $\mathrm{i}$ & - & alf & $\mathrm{b}$ & $\mathrm{R}$ \\
\hline
\end{tabular}

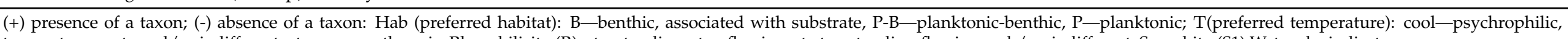

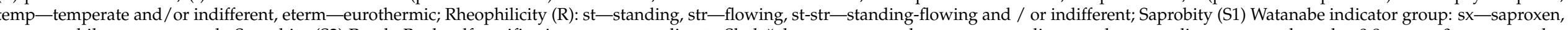

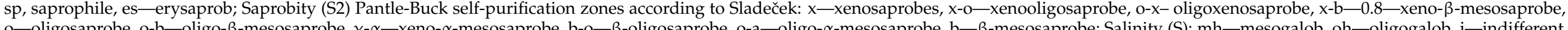

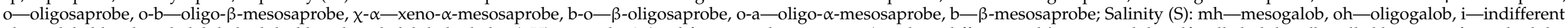

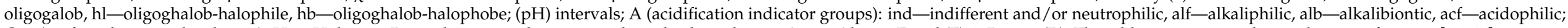
Geo (preferred geographical zone): Ha—Holarctic, Pt—palaeotropic, k—cosmopolitan, b—boreal, a-a—Arctic-Alpine; Fossil (F) \& Recent (R). The table was generated according to references [37-45]. 


\subsection{Diatom Biostratigraphy}

The total amount of diatom valves in the core varies with depth from 0.02 to $55.5 \mathrm{mln}$ valves / $g$ of air-dried sediment with several maxima at the depths of $41.0-41.5 \mathrm{~m}(55.5-42.1 \mathrm{mln}$ valves/g), $38.0 \mathrm{~m}$ (39.7 mln valves/g), and $19.5 \mathrm{~m}$ (41.0 mln valves/g) (Figure 4$)$. On the basis of data on total concentration of diatom valves, taxonomic diversity, and ecological characteristics of the species (Table 1), two diatom ecozones were established. Ecozone I (49.5-35.0 m) contains 87 species, the abundance of which varies from 0.3 to $55.5 \mathrm{mln}$ valves/g of dry sediment.

The lower part of the core (49.5-47.5 m) is dominated by planktonic-benthic and benthic diatoms Staurosira construens Ehrenberg; Pseudostaurosira elliptica (Schumann) Edlund, E. Morales \& Spaulding; Punctastriata lancettula (Schumann) P.B. Hamilton \& Siver; Staurosira binodis (Ehrenberg) Lange-Bertalot; Staurosira construens var. triundulata (Reichelt) Bukhtiyarova and Staurosira leptostauron (Ehrenberg) Kulikovskiy \& Genkal, the abundance of which varies from 1.4 to $23.5 \mathrm{mln}$ valves $/ \mathrm{g}$.

Up along the core, in the interval of 47.0-35 m, the concentration of valves of benthic diatoms decreased, and those of planktonic ones increased. In the depth intervals of 41-42 m (16.8-55.5 mln valves/g) and of 37.5-38.5 m (14.4-39.7 mln valves/g) (Figure 4), planktonic diatoms are mainly represented by the species Aulacoseira ambigua (Grunow) Simonsen and $A$. ambigua f. curvata (Skabichevskij) Genkal, with maximum abundances of 10.5 and $29.3 \mathrm{mln}$ valves/g, respectively. Other planktonic diatoms occurred in small amounts; these are endemics Cyclotella tuncaica Nikiteeeva, Likhoshway \& Pomazkina; Aulacoseira aff. baicalensis (Wislouch) Simonsen; Stephanodiscus tuncaensis Pomazkina \& Likhoshway and Stephanodiscus sp. (Figures 4 and 5). Among planktonic-benthic diatom algae in the Ecozone I, the species Melosira undulata (Ehrenberg) Kützing; Ellerbeckia arenaria f. teres (Brun) R.M. Crawford; Aulacoseira distans (Ehrenberg) Simonsen; Planothidium lanceolatum (Brébisson ex Kützing) Lange-Bertalot and Gomphonema angustatum (Kützing) Rabenhorst (Table 1; Figures 4 and 5) occurred. The benthic assemblage is rather diversified (Figure 4; Table 1). The total amount of benthic species in sediments varies from 0.05 to $10.5 \mathrm{mln}$ valves/g. The samples at depths $44.5,44.0,38.5$, and $38.0 \mathrm{~m}$, which had the highest diversity in the number of benthic species (from 12-18 species), were characterised by Achnanthidium minutissimum (Kützing) Czarnecki; Amphora aff. inariensis Krammer; A. ovalis (Kützing) Kützing; Cavinula jaernefeltii (Hustedt) D.G. Mann \& A.J. Stickle; C. scutiformis (Grunow) D.G. Mann \& A.J. Stickle; Cymbella aff. laevis Nägeli; Cymbopleura cuspidate (Kützing) Krammer; Diploneis elliptica (Kützing) Cleve; D. parma Cleve; Eolimna aboensis (Cleve) S.I. Genkal; Epithemia gibba (Ehrenberg) Kützing; Eunotia minor (Kützing) Grunow; E. robusta Ralfs; E. tenella (Grunow) Hustedt; Fragilaria aequalis Heiberg; F. capucina Desmazières; Gomphonella olivacea (Hornemann) Rabenhorst; Hippodonta costulata (Grunow) Lange-Bertalot, Metzeltin \& Witkowski; Navicula gregaria Donkin; N. radiosa Kützing; N. viridula (Kützing) Ehrenberg; Odontidium mesodon (Kützing) Kützing; Pinnularia abaujensis var. linearis (Hustedt) R.M. Patrick; P. brebissonii (Kützing) Rabenhorst; P. microstauron (Ehrenberg) Cleve; Placoneis gastrum (Ehrenberg) Mereschkowsky; Planothidium joursacense (Héribaud) Lange-Bertalot; P. linkei (Hustedt) Lange-Bertalot; Pseudostaurosira elliptica (Schumann) Edlund; Sellaphora bacillum (Ehrenberg) D.G. Mann; Surirella librile (Ehrenberg) Ehrenberg; Staurosira construens var. triundulata; S. leptostauron (Ehrenberg) Kulikovskiy; S. tabellaria (W. Smith) Leuduger-Fortmorel; Staurosirella pinnata (Ehrenberg) D.M. Williams \& Round; Tetracyclus ellipticus var. latissimus Hustedt and T. glans (Ehrenberg) F.W. Mills. 


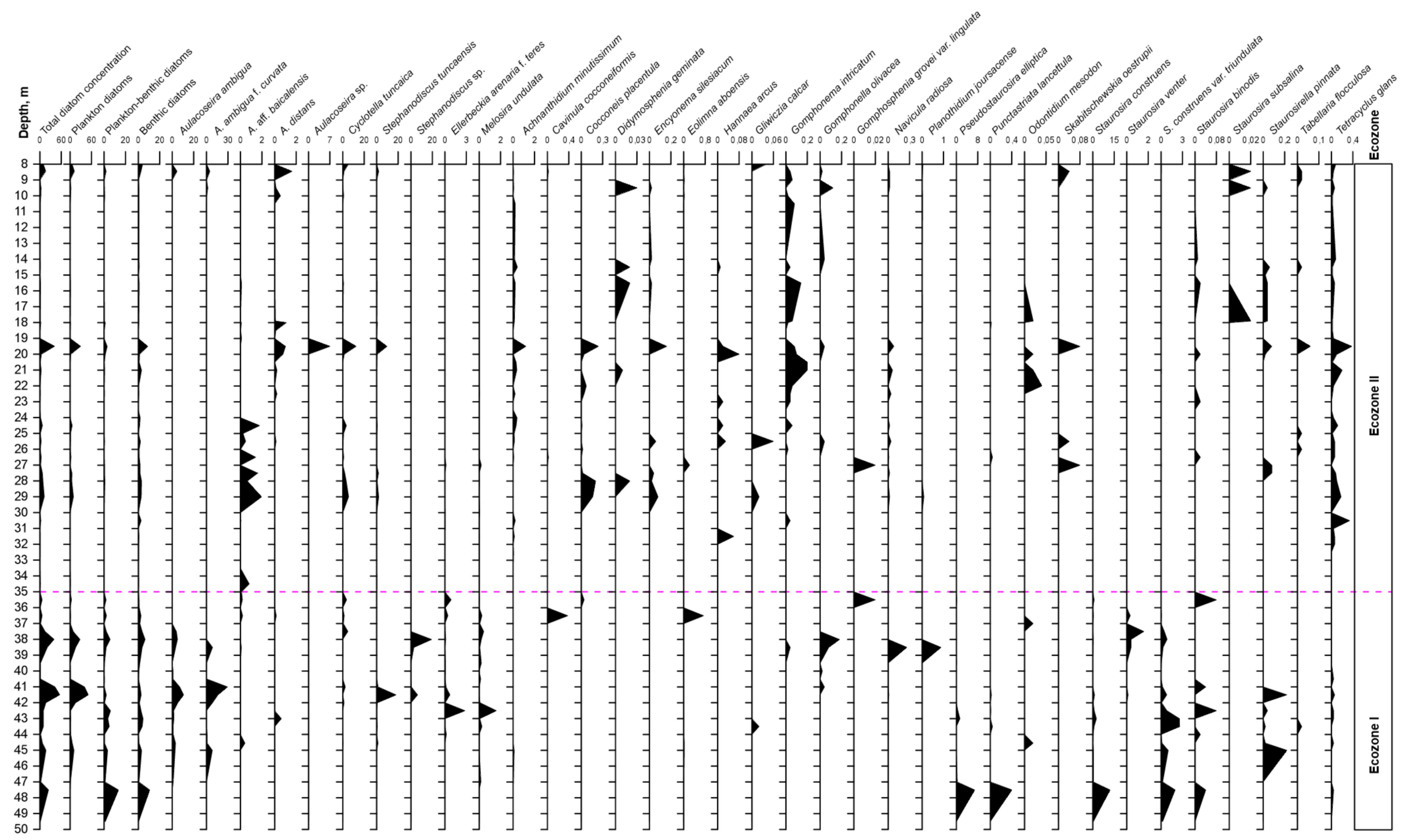

Figure 4. Diatom diagram from core Tunka-13, showing the succession of the most common taxa (valves, mln/g air-dried sediment). 


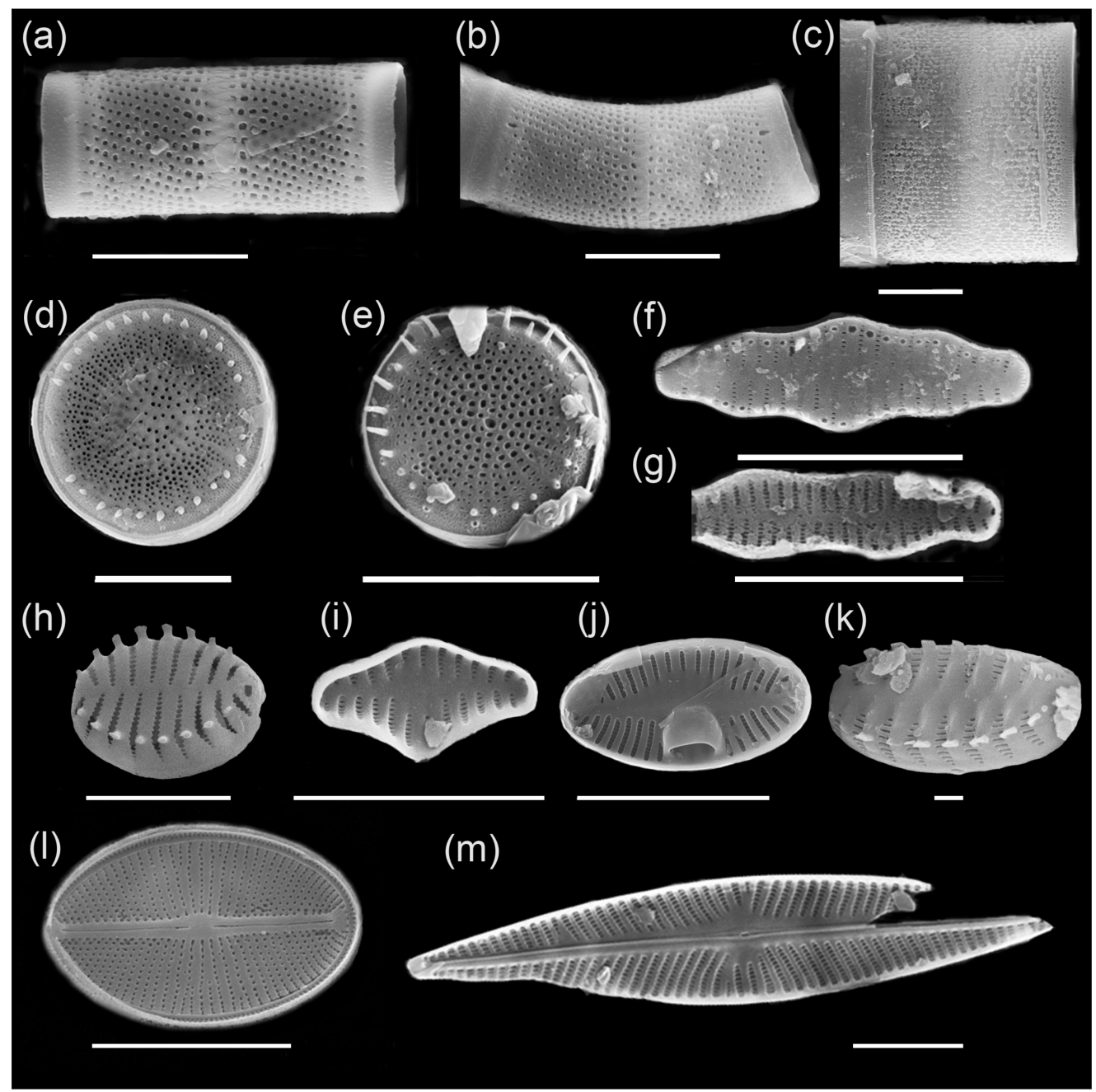

Figure 5. Main complex of diatoms of Ecozone I: (a) Aulacoseira ambigua, (b) A. ambigua f. curvata, (c) Melosira undulata, (d) Stephanodiscus tuncaensis, (e) Stephanodiscus sp., (f) Staurosira construens var. triundulata, (g) Staurosira binodis, (h) Staurosira venter, (i) Staurosira construens, (j) Planotidium joursacense, (k) Staurosirella pinnata, (1) Cavinula cocconeiformis, (m) Navicula radiosa. Scale bars: $10 \mu \mathrm{m}(\mathbf{a}-\mathbf{j}, \mathbf{l}, \mathbf{m})$ and $1 \mu \mathrm{m}(\mathbf{k})$.

Ecozone II (35.0-8.0 m) differs from Ecozone I by having lower values of diatom valve concentration and by having a different taxonomic composition (Figures 4 and 6; Table 1). A total of 143 species are found here. According to the graph of total abundance of diatoms, one can notice three main peaks: in the depth's interval of $29 \mathrm{~m}, 19 \mathrm{~m}$, and $8.45 \mathrm{~m}$, with a maximum abundance of $12.2 \mathrm{mln}$ valves $/ \mathrm{g}$, $41.0 \mathrm{mln}$ valves $/ \mathrm{g}$, and $16.3 \mathrm{mln}$ valves/g, respectively.

The dominant complex of the first type included planktonic diatoms Cyclotella tuncaica and Aulacoseira aff. baicalensis. Maximal values of their abundance are found at the depth of $29 \mathrm{~m}$ and were 5.42 and $1.96 \mathrm{mln}$ valves/g, respectively. Other planktonic species Stephanodiscus tuncaensis occurred in the interval of $29.0-24.5 \mathrm{~m}$ in amounts up to $1.8 \mathrm{mln}$ valves $/ \mathrm{g}$, and at the depth of $19.5 \mathrm{~m}$, the maximum reached values $9.6 \mathrm{mln}$ valves $/ \mathrm{g}$. At the same horizon $(19.5 \mathrm{~m})$, a high abundance $(7.0 \mathrm{mln}$ valves/g) of Aulacoseira sp. (Figure $6 \mathrm{f}$ ) and Aulacoseira distans ( $1.0 \mathrm{mln}$ valves/g) was noticed. The abundance of Cyclotella tuncaica reached its maximum values of $12.4 \mathrm{mln}$ valves $/ \mathrm{g}$. The third peak is characterised by small values of planktonic diatoms Aulacoseira ambigua (4.4 mln valves/g), Aulacoseira ambigua $\mathrm{f}$. curvata (4.6 mln valves/g), Cyclotella tuncaica ( $5.2 \mathrm{mln}$ valves/g), and of the planktonic-benthic species Aulacoseira distans ( $1.6 \mathrm{mln}$ valves/g). 


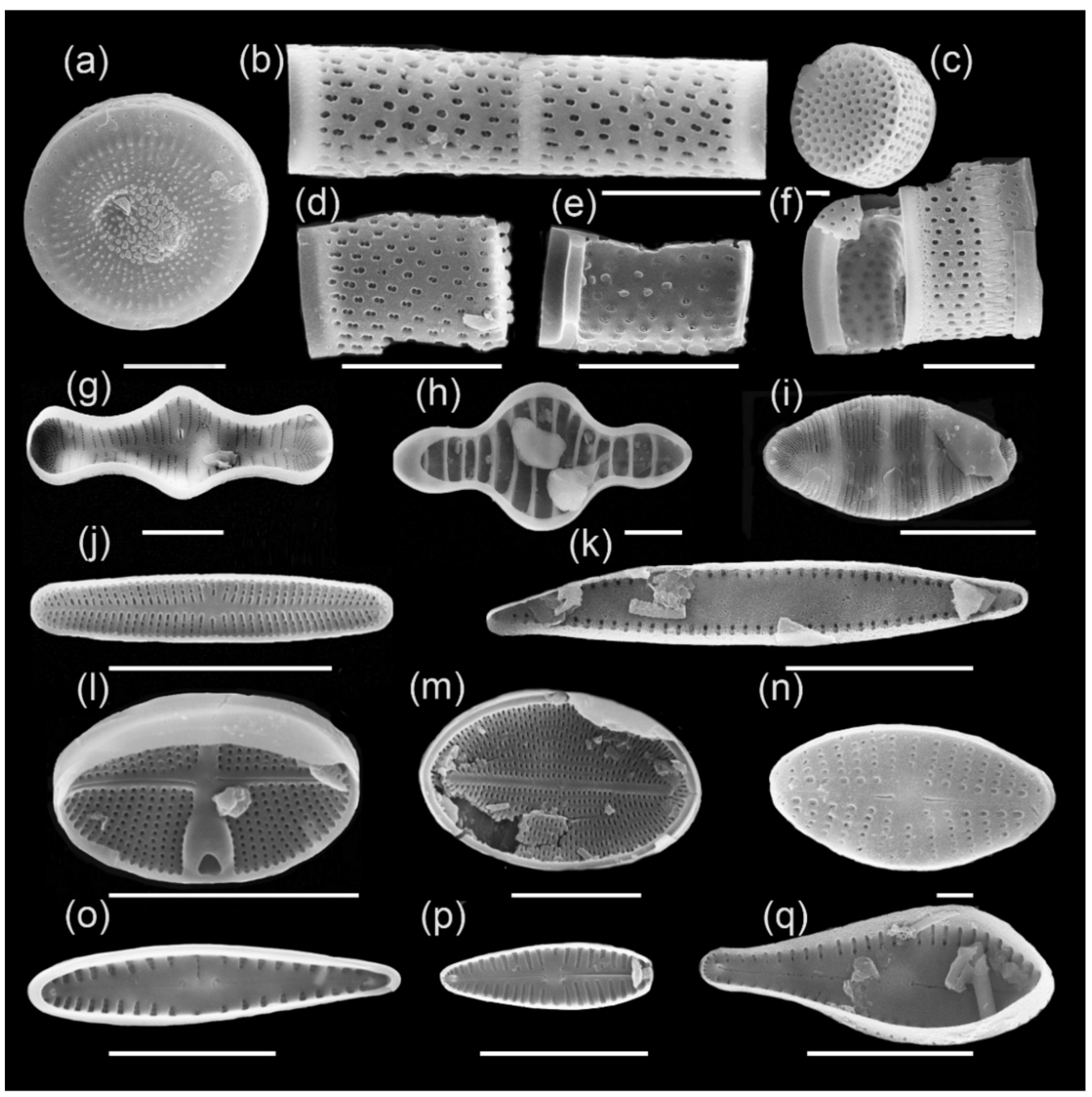

Figure 6. Main complex of diatoms of Ecozone II: (a) Cyclotella tuncaica, (b,d,e) Aulacoseira aff. baicalensis, (c) Aulacoseira distans, (f) Aulacoseira sp., (g) Tabellaria flocculosa, (h) Tetracyclus glans, (i) Odontidium mesodon, (j) Achnanthidium minutissimum, (k) Staurosira subsalina, (1) Gliwiczia calcar, (m) Cocconeis placentula, (n) Eolimna minima, (o) Gomphonema intricatum, (p) Gomphonella olivacea, (q) Gomphosphenia grovei var. lingulata. Scale bars: $10 \mu \mathrm{m}(\mathbf{a}, \mathbf{b}, \mathbf{d}-\mathbf{m}, \mathbf{o}-\mathbf{q})$ and $1 \mu \mathrm{m}(\mathbf{c}, \mathbf{n})$.

The abundance of planktonic-benthic diatoms in this interval $(35-8 \mathrm{~m})$ varies from 0 to $2.9 \mathrm{mln}$ valves $/ \mathrm{g}$. The following species were recorded: Planothidium lanceolatum; Gomphonema intricatum Kützing; Pseudostaurosira brevistriata (Grunow) D.M. Williams \& Round; Staurosirella martyi (Héribaud) E. Morales \& K.M. Manoylov; Ulnaria ulna (Nitzsch) Compère; Navicula cryptocephala Kützing; Stauroneis anceps Ehrenberg; Stauroneis smithii Grunow; Pinnularia viridis (Nitzsch) Ehrenberg; Tabellaria fenestrata (Lyngbye) Kützing; Tabellaria flocculosa (Roth) Kützing; Tetracyclus emarginatus (Ehrenberg) W. Smith and Cocconeis placentula Ehrenberg (Table 1).

The abundance of benthic diatoms in the Ecozone II varies from 0 to $8.8 \mathrm{mln}$ valves $/ \mathrm{g}$. Such species occurred here often, such as Achnanthidium minutissimum; Didymosphenia geminata (Lyngbye) Mart. Schmidt; Encyonema silesiacum (Bleisch) D.G. Mann; Hannaea arcus Ehrenberg; Gliwiczia calcar (Cleve) M. Kulikovskiy, Lange-Bertalot \& A. Witkowski; Gomphonella olivacea; Gomphosphenia grovei var. lingulate (Hustedt) Lange-Bertalot; Navicula radiosa; Odontidium mesodon, Skabitscherwskia oestrupii (A. Cleve) Kulikovskiy \& LangeBertalot; Staurosira binodis, Staurosira subsalina (Hustedt) Lange-Bertalot; Staurosirella pinnata and Tetracyclus glans (Figures 4 and 6; Table 1). 
Ecozone II differs in its greater taxonomic diversity compared to Ecozone I (143 species vs. 87). The diversity increase occurred due to a greater number of planktonic-benthic and benthic taxa.

\subsection{Ecological Analysis of Diatom Flora}

Diatom species have been grouped on the basis of their known ecological preferences and geographic distribution. This allowed us to better distinguish the main difference between Ecozones I and II (Figure 7).

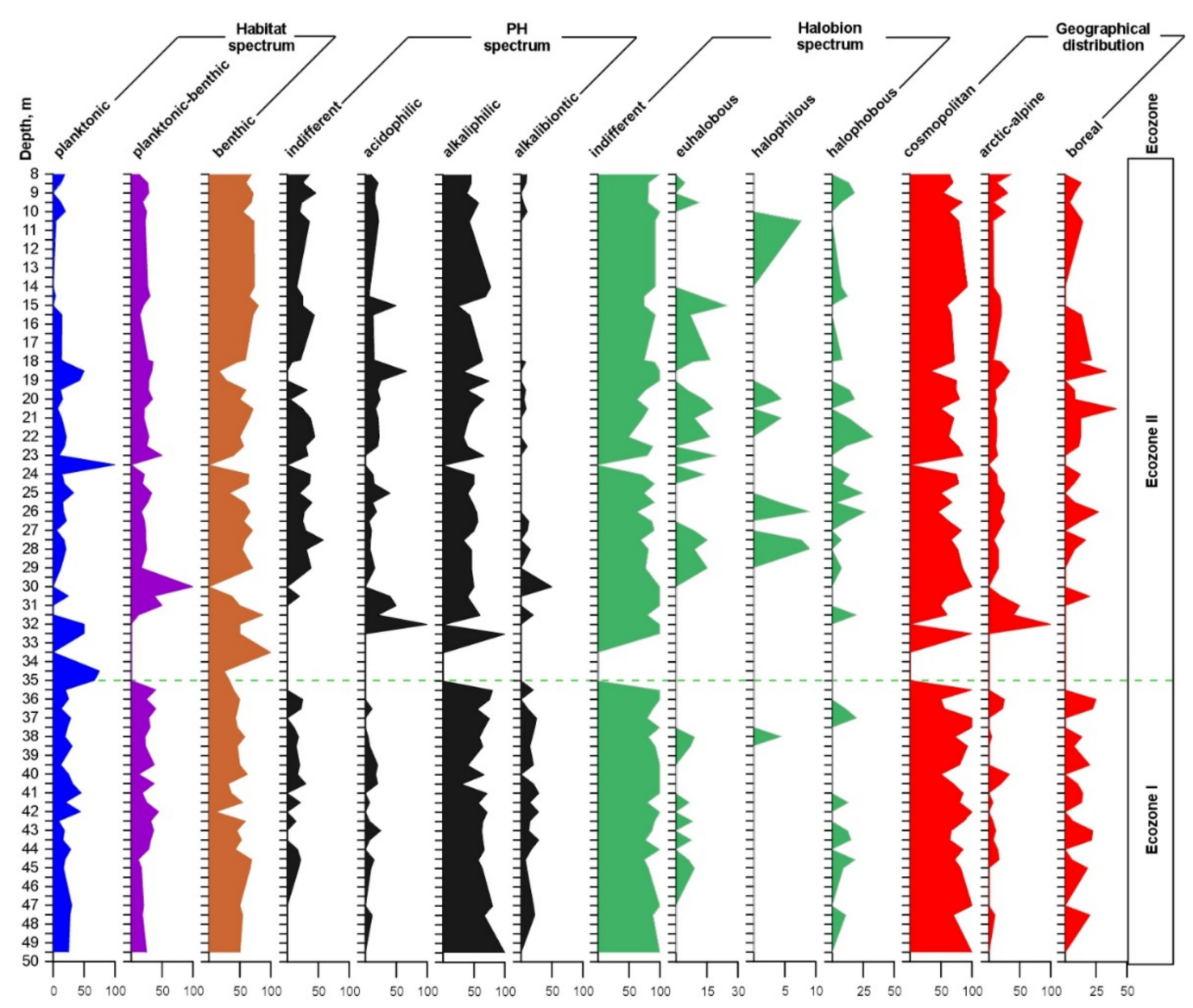

Figure 7. Different diatom ecological groups in the core Tunka-13, along the xaxis (\%), and along the y axis (core depth, $\mathrm{m}$ ).

Habitat spectrum shows that the number of benthic taxa is dominant in the whole core, except the horizons of 40.5, 41.0, and $42.0 \mathrm{~m}$ in Ecozone I and 35.0-35.5, 30.0, 23.5-25.0, and $18.5-19.0 \mathrm{~m}$ in Ecozone II, where peaks of development are noticed for planktonic and planktonic-benthic taxa. Abrupt changes of the planktonic diatom amount suggest changes in environmental conditions and in water body depth.

In relation to the $\mathrm{pH}$ of the water, the content of alkaliphilic ranged between 25 and $100 \%$, followed by indifferent $7-58 \%$, acidophilic $6-67 \%$, and alkalibiontic $4-50 \%$. Alkaliphilic diatoms dominate along the whole core. Indifferent and acidophilic species numbers were higher in Ecozone II, while the number of alkalibiontic forms was lower (Figure 7).

According to the halobium system, the indifferent oligohalobous taxa predominate, ranging from 50 to $100 \%$. Halophobous taxa account for $0-27 \%$. The distribution of these two groups was more or less even, while two other groups (euhalobous and halophilous) dominated in Ecozone II.

In terms of biogeographic distribution, the abundance of cosmopolitan forms predominated (33-92\%), followed by boreal (0-25\%). The spectrum of the arctic-alpine species ranged between 0 and $33 \%$, except the horizons of 32-31 m, where their amount reached 
40-100\% (Figure 7). It is shown that in Ecozone II, there were more arctic-alpine species than in Ecozone I.

Among all identified diatoms, 116 species and forms, $68.2 \%$ of the whole assemblage, are useful indicators of organic matter enrichment. The fraction of o-saprobes was $23 \%$, o- $\beta$-mesosaprobes was $16 \%$, x-o-saprobes was $13 \%$, o- $\alpha$-mesosaprobes was $9 \%$, $x-$ xenosaprobes and $\beta$ - $\alpha$-mesosaprobes were $8 \%$ each, $\beta$-mesosaprobes, $\alpha-\beta$-mesosaprobes, and $x-\beta$-mesosaprobionts were $5 \%$ each, and o-xand $\beta$-o-saprobes were $4 \%$ each. There were no polysaprobes. Indicators of pure water xeno $(\chi, \chi-0)$ - and oligosaprobes $(0-\chi, \chi-\beta$, $0,0-\beta$ ) represent $69 \%$ of the indicator species as a whole.

Temperature ranges have been inferred by means of 36 species, 19 of them occurring under moderate temperature conditions, of which 12 are psychrophilic, three are eurythermic, and two are thermophilic. There were data on rheophilicity for only 47 diatom species, 30 of them characteristic of standing-current waters, 10 of current waters (rivers and creeks), and 7 were characteristic for standing waters.

Thus, ecological-geographic analysis of diatoms showed dominance of benthic, alkaliphilic, indifferent, cosmopolitan, and oligosaprobe species and representatives of standing-current waters. It is shown that during the period corresponding to sedimentation in Ecozone II, the water body was cooler, as suggested by the increase of arctic-alpine taxa.

\section{Discussion}

Previously, it was shown that elevated concentrations of diatoms in the Lake Baikal palaeorecord reflect warm periods $[2,46]$. This study shows that in the depth interval of 49.5-37.5 m (Ecozone I), diatom valve content was the highest, suggesting warmer and more humid climatic conditions during the Early Pliocene (5.33-3.6 Ma). This period was marked by short but rather well-expressed warming and by smoothing of contrasts between winter and summer monsoons due to decrease of intensity and duration of the former [47]. The second half of the Pliocene (3.6-2.5 Ma) was characterised by an intensive and strong cooling [2,47-50]. This cooling is reflected along the section of the hole Tunka-13 in the depth interval of 35.0-8.0 m (Ecozone II), where diatom concentration decreases abruptly. Dominant genera change from Aulacoseira to Stephanodiscus and Cyclotella.

We can see correlation between our data and a Baikal core BDP-96-1 (Figure 5.2. in [2]) (p. 44), where the amount of Aulacoseira decreases abruptly in the interval of 3.8-3.2 Ma, and then the endemic representatives of the genera Stephanodiscus and Cyclotella dominate. In the core Tunka-13 the decrease of Aulacoseira occurs above $35 \mathrm{~m}$. Here also the number of arctic-alpine taxa increases.

Besides species diversity level, Ecozones I and II differ by diatom taxonomic composition. Sixty species occur in both zones, 27 characteristic only of Ecozone I and 83 only of Ecozone II (Figure 8).

In order to compare the complex of species from the core Tunka-13 (Ecozone I and II) with the Tunka Pliocene complexes described in previous works [6], only the common species have been included in the Venn diagram. A total of 51 common species have been highlighted, 24 occurring both in Ecozone I and II, 33 common to Ecozone I and Tunka, and 42 common to Ecozone II and Tunka.

Unfortunately, we cannot compare the fossil benthic taxa from the Pliocene deposits of Lake Baikal and the Tunka-13 core. There are no data on the species composition of benthic diatoms in the Baikal palaeochronicle. It is known that these are representatives of the genera Staurosira, Staurosirella, Achnanthidium, Planothidium, Eunotia, Amphora, Navicula, Cavinula, Stauroneis, Pinnularia, Gomphonema, Cymbella, etc. [2]. The same genera are found in the core Tunka-13. Since the majority of benthic species from the core Tunka-13 are extant, we compared them with data on Lake Baikal phytobenthos [31,34,35,51]. It was shown that among 170 species from the core Tunka-13, 85 (50\%) occur in Lake Baikal phytobenthos. There is a great similarity with diatoms from Ecozone II, where 75 common species are found, while in Ecozone I there are 44. 


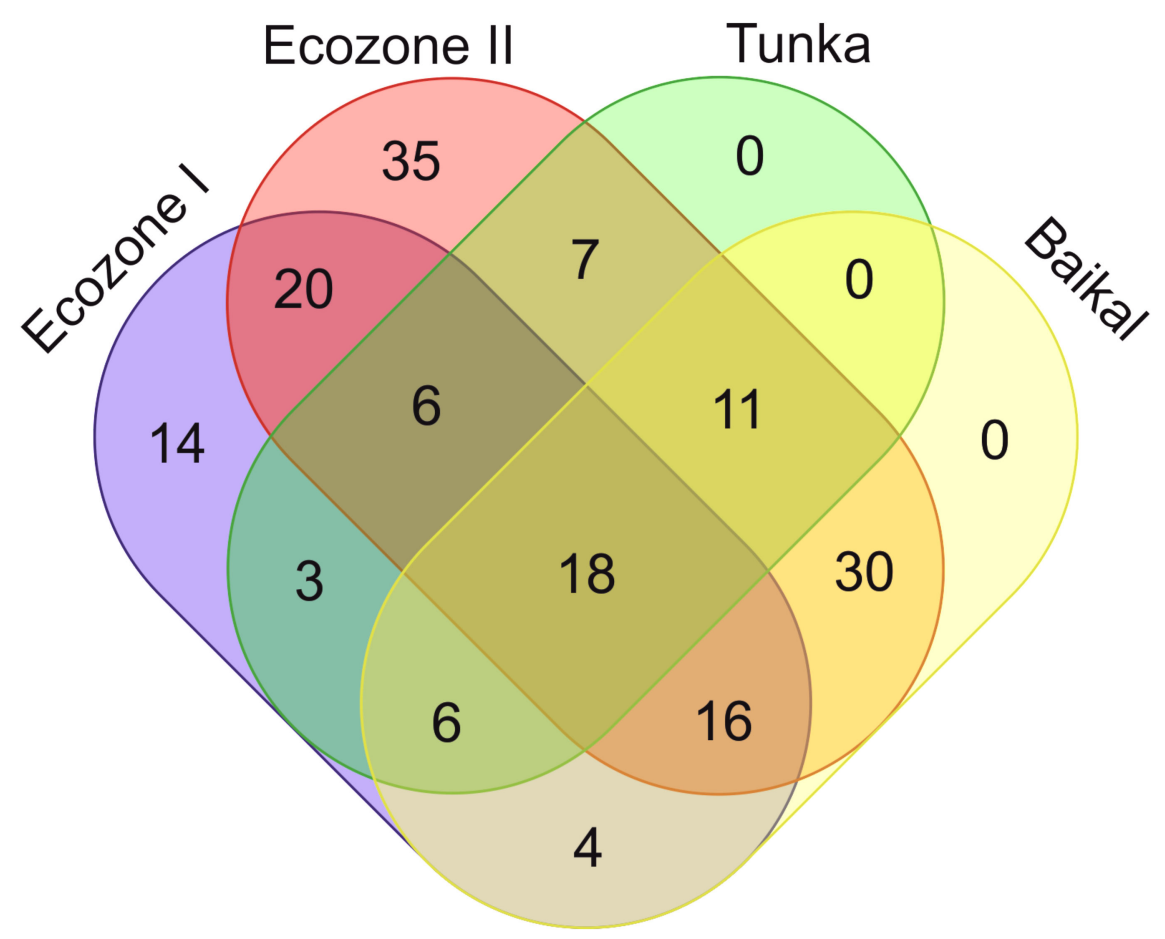

Figure 8. Venn diagram illustrating the number of common species between diatoms from Tunka-13 (Ecozone I, Ecozone II), Pliocene sediments in Tunka (by data from [6]), and modern phytobenthos from Lake Baikal [31,34,35,51].

According to previous work studying cores from the holes in the area of Akhalik deposit of Tunka Depression [6], it is shown that in Pliocene sediments, forms of shallowwater lakes and near-shore zones are replaced by complexes where planktonic diatoms dominate. In the core Tunka-13, there are also several diatom successions, according to which a palaeolake can be characterised during different time intervals. Initially (depths interval of 49.5-47.2 m), a palaeoreservoir was shallow. Benthic taxa dominated. Later (47.0-41.0 m) the palaeoreservoir deepened (up to several tens of metres), and planktonic diatoms Aulacoseira ambigua and Aulacoseira ambigua f. curvata started to appear and dominate the diatom assemblages. These species are also found in Upper MiocenePliocene Baikal deposits [2]. In modern Lake Baikal there are no A. ambigua f. curvata, and A. ambigua prefers well-warmed bays and shallow-water areas and develops in summerautumn phytoplankton at water temperature $12-20{ }^{\circ} \mathrm{C}$ [52]. Further $(40.5-39.5 \mathrm{~m})$, the abundance of Aulacoseira considerably decreased, probably in response to the palaeolake depth reduction. Subsequent increase of the abundance of planktonic species occurred in the depth's interval of 38.5-37.5 m, suggesting reestablishment of hydrological conditions. Further (35.0-8.0 m), an abrupt decrease of diatom valve concentration and change of dominant species complex are observed. Cyclotella tuncaica becomes dominant. There are small amounts of Aulacoseira aff. baicalensis.

A. baicalensis is a pelagic psychrophilic species that develops in Lake Baikal in spring during ice-cover period and immediately after ice breakup on the lake [52]. It is known in the Lake Baikal palaeorecord from $122 \mathrm{ky}$ BP [53]. At present, in the phytoplankton of Lake Baikal, this species has a wide morphological variability of valves [54], but its variability in the fossil record is unknown. It occurred in the Pliocene in Tunka Valley [6,7], on Vitim Plateau $[6,55,56]$. There is also information on the absence of $A$. baicalensis in Upper Miocene sediments of Tunka Valley [10]. In this paper, we mark valves similar to this species as Aulacoseira aff. baicalensis, as we need detailed studies of the ultrastructure of numerous valves of this species from different locations, and this is a subject of further study. 


\section{Conclusions}

As a result of taxonomic analysis, 170 species of diatoms were identified. Their distribution in core Tunka-13 is highly variable and reflects the instability of the hydrological regime and sedimentary environment, which is confirmed by the lithological features of the section. The ecological composition of diatoms indicates a large area of the littoral zone of the palaeoreservoir, despite the deepening of the palaeolake at certain stages. In terms of taxonomic diversity and ecology of species, two ecozones are distinguished. Ecozone I differs from Ecozone II by a higher concentration of diatom valves, lower taxonomic diversity, and a large number of boreal species. This indicates the formation of deposits in the warmer climate of the Early Pliocene.

The occurrence of planktonic local endemic species Cyclotella tuncaica and Stephanodiscus tuncaensis indicates the isolation of the palaeoreservoir from Lake Baikal, despite geographical proximity. In addition, we did not find representatives of the endemic Baikal genera Stephanopsis or Tertiariopsis, widely developing in the Pliocene. Based on the data obtained, we infer that in the Pliocene, the palaeoreservoir was isolated in the Tunka Basin. There was no transgression of water from Lake Baikal into the Tunka palaeoreservoir or, conversely, there was no penetration of diatom species into the water of Lake Baikal along rivers.

Author Contributions: Conceptualisation, M.U. and A.H.; collected samples, M.U. and L.T.; microscopy and data analysis, M.U., E.R., and L.T.; statistical analysis, I.M.; funding Acquisition, M.U.; interpretation of results, L.T., M.U., E.R., and A.H.; project administration, M.U.; writing-original draft preparation, M.U., A.H., and L.T.; writing—review and editing, M.U., A.H., L.T., E.R., and S.R. All authors have read and agreed to the published version of the manuscript.

Funding: The investigation was performed with financial support of the State Assignments of the Limnological Institute of the Siberian Branch of the Russian Academy of Sciences \# 0279-2021-0008 (121032300186-9) and the RFBR Grant \#19-04-00746.

Institutional Review Board Statement: Not applicable.

Data Availability Statement: Not applicable.

Acknowledgments: The authors are thankful to A.G. Kazarin, Director of KASKAD-M LLC, for hole drilling, Yu. Kaplukova for English translation, Ye. Likhoshway for helpful suggestions and comments, and the anonymous reviewers for their contributions to a previous version. The study was performed using microscopes of the Instrumental Centre "Electron Microscopy" (http: / /www.lin.irk.ru/copp/) of the Shared Research Facilities for Research "Ultramicroanalysis".

Conflicts of Interest: The authors declare no conflict of interest.

\section{References}

1. Mats, V.D.; Ufimtsev, G.F.; Mandelbaum, M.M. The Cenozoic Baikal Rift Basin: Its Structure and Geological History; Academic Publishing House Geo: Novosibirsk, Russia, 2001; p. 252. (In Russian)

2. Kuz'min, M.I.; Khursevich, G.K.; Prokopenko, A.A.; Fedenya, S.A.; Karabanov, E.B. Late Cenozoic Centric Diatoms of Lake Baikal; Academic Publishing House Geo: Novosibirsk, Russia, 2009; p. 370. (In Russian)

3. Logachev, N.A.; Zorin, Y.A. Baikal Rift Zone: Structure and geodynamics. Tectonophysics 1992, 208, 273-286. [CrossRef]

4. Rasskazov, S.V. Magmatism of the Baikal Rift System; Nauka: Novosibirsk, Russia, 1993; p. 288. (In Russian)

5. Chuvashova, I.S.; Rasskazov, S.V. Sources of Magmatism in the Mantle of the Evolving Earth; ISU Publishing House: Irkutsk, Russia, 2014; p. 291. (In Russian)

6. Cheremisinova, E.A. Diatom Flora of Neogene Sediments in Pribaikalie; Nauka: Novosibirsk, Russia, 1973; p. 83. (In Russian)

7. Popova, S.M.; Mats, V.D.; Chernyaeva, G.P.; Shimaraeva, M.K.; Kul'chitsky, A.A.; Vorobyova, G.A.; Klimanova, V.M.; Kononov, Y.; Kravchinsky, A.Y.; Kulagina, N.V.; et al. Baikal Rift Zone: Paleolimnological Reconstructions; Nauka: Novosibirsk, Russia, 1989; p. 111. (In Russian)

8. Lupikina, E.G.; Khursevich, G.K. New genus of the class Centrophyceae (Bacillariophyta). Algologiya 1991, 1, 67-70.

9. Khursevich, G.K. Morphology and taxonomy of some centric diatom species from the Miocene sediments of the Dzhilinda and Tunka hollow. Mem. Calif. Acad. Sci. 1994, 17, 269-280.

10. Likhoshway, E.V.; Pomazkina, G.V.; Nikiteeva, T.A. Centric diatoms from the Miocene deposits of the Baikal Rift Zone (Tunka depression). Russ. Geol. Geophys. 1997, 38, 1445-1452. 
11. Hassan, A.; Rasskazov, S.V.; Chuvashova, I.S.; Yasnygina, T.A.; Titova, L.A.; Kulagina, N.V.; Usoltseva, M.V. Identifying Upper Miocene-Lower Pliocene lacustrine sediments in dry Tunkabasin of the Baikal Rift Zone. Geodyn. Tectonophys. 2020, 11, 262-284. [CrossRef]

12. Rasskazov, S.V.; Lyamina, N.A.; Chernyaeva, G.P.; Luzina, I.V.; Rudnev, A.F.; Rezanov, I.N. Cenozoic Stratigraphy of the Vitim Plateau: Phenomenon of Long-Term Rifting in the South of East Siberia; Academic Publishing House Geo: Novosibirsk, Russia, 2007; p. 193. (In Russian)

13. Logatchev, N.A. Cenozoic continental deposits in the Baikal type basins. Izv. AN SSSR 1958, 4, 18-29. (In Russian)

14. Grachev, M.A.; Likhoshvay, E.V.; Vorobyova, S.S.; Khlystov, O.M.; Bezrukova, E.V.; Veinberg, E.V.; Goldberg, E.L.; Granina, L.Z.; Kornakova, E.G.; Lazo, F.I.; et al. Paleoclimate signals of the Upper Pleistocene in sediments of Lake Baikal. Geol. Geophys. 1997, $38,957-980$

15. Skvortzow, B.W.; Meyer, C.I. A Contribution to the diatoms of Baikal Lake. Proc. Sungaree River Biol. Stn. 1928, 1, 1-55.

16. Skvortzow, B.W. Bottom diatoms from Olhongate of Lake Baikal. Philipp. J. Sci. 1937, 62, $293-377$.

17. Gleser, S.I.; Jousé, A.P.; Makarova, I.V.; Proschkina-Lavrenko, A.I.; Sheshukova-Poretzkaya, V.S. The Diatoms of the USSR Fossil and Recent; Nauka: Leningrad, Russia, 1974; p. 403. (In Russian)

18. Houk, V. Atlas of Freshwater Centric Diatoms with a Brief Key and Descriptions: Part. I. Melosiraceae, Orthoseiraceae, Paraliaceae and Aulacoseiraceae; Fottea: Olomouc, Czech Republic, 2003; pp. 1-27.

19. Houk, V.; Klee, R. Atlas of Freshwater Centric Diatoms with a Brief Key and Descriptions: Part. II. Melosiraceae and Aulacoseiraceae; Fottea: Olomouc, Czech Republic, 2007; pp. 85-255.

20. Kozyrenko, T.; Strelnikova, N.; Khursevich, G.; Tsoy, I.; Yakovschikova, T.; Mukhina, V.; Olshtynskaja, A.; Semina, G. The Diatoms of Russia and Adjacent Countries Fossil and Recent II; Strelnikova, N., Tsoy, I., Eds.; St. Petersburg University Press: St. Petersburg, Russia, 2008; p. 171. (In Russian)

21. Gleser, S.I.; Makarova, I.V.; Moisseeva, A.I.; Nokolaev, V.A. (Eds.) The Diatoms of the USSR. Fossil and Recent; Nauka: Leningrad, Russia, 1988; Volume 2, Issue 1, p. 116. (In Russian)

22. Gleser, S.I.; Makarova, I.V.; Moisseeva, A.I.; Nokolaev, V.A. (Eds.) The Diatoms of the USSR. Fossil and Recent; Nauka: St. Petersburg, Russia, 1992; Volume 2, Issue 2, p. 125. (In Russian)

23. Loseva, E.I. Atlas of the Late Pliocene Diatoms of River Kama Region; Nauka: Leningrad, Russia, 1982; p. 204. (In Russian)

24. Krammer, K.; Lange-Bertalot, H. Bacillariophyceae. Teil 1: Naviculaceae, Bd. 2/1: Süßwasser flora von Mitteleuropa; Jena VEB Gustav Fischer Verlag: Stuttgart, Germany, 1986; p. 876.

25. Lange-Bertalot, H.; Krammer, K. Achnanthes eine Monographie der gattungmit Definition der Gattung Cocconeis und Nachträgenzu den Naviculaceae; Bibl. Diatom: Berlin, Germany; Stuttgart, Germany, 1989; p. 385.

26. Lange-Bertalot, H. Diatoms of Europe: Navicula sensu stricto, 10 Genera Separated from Navicula sensu lato Frustulia; Gantner, A.R.G., Ed.; Ruggell: Lichtenstein, Germany, 2001; p. 526.

27. Krammer, K. Die Cymbelloid en Diatomeen. Teil 2. Encyonema part., Encyonopsis and Cymbellopsis; Bd. 37; Bibl. Diatom: Berlin, Germany; Stuttgart, Germany, 1997; p. 469.

28. Krammer, K. Diatoms of Europe: The genus Pinnularia; Gantner, A.R.G., Ed.; Ruggell: Lichtenstein, Germany, $2000 ;$ p. 703.

29. Krammer, K. Diatoms of Europe: Cymbella; Gantner, A.R.G., Ed.; Ruggell: Lichtenstein, Germany, $2002 ;$ p. 584.

30. Krammer, K. Diatoms of Europe: Cymbopleura, Delicata, Navicymbula, Gomphocymbellopsis, Afrocymbella; Gantner, A.R.G., Ed.; Ruggell: Lichtenstein, Germany, 2003; p. 530.

31. Kulikovskiy, M.S.; Lange-Bertalot, H.; Metzeltin, D.; Witkowski, A. Diatoms of Europe: Lake Baikal: Hotspot of Endemic Diatoms I; Gantner, A.R.G., Ed.; Ruggell: Lichtenstein, Germany, 2012; Volume 23, p. 861.

32. Kulikovskiy, M.S.; Lange-Bertalot, H.; Kuznetsova, I.V. Iconograph Diatom: Lake Baikal: Hotspot of Endemic Diatoms II; Gantner, A.R.G., Ed.; Ruggell: Lichtenstein, Germany, 2015; Volume 26, p. 656.

33. Kulikovskiy, M.S.; Glushchenko, A.M.; Genkal, S.I.; Kuznetsova, I.V. Identification Book of Diatoms from Russia; Dorofeyuk, N.I., Ed.; Filigran: Yaroslav, Russia, 2016; p. 804. (In Russian)

34. Pomazkina, G.B.; Rodionova, E.V. Diatoms of the Family Cymbellaceae of Lake Baikal: Atlas and Key; Nauka: Novosibirsk, Russia, 2014; p. 242. (In Russian)

35. Pomazkina, G.B.; Rodionova, E.V.; Sherbakova, T.A. Benthic Diatoms of the Family Naviculaceae of Lake Baikal: Atlas and Key; Nauka: Novosibirsk, Russia, 2018; p. 315. (In Russian)

36. Guiry, M.D.; Guiry, G.M. AlgaeBase. 2021. Available online: http:/ / www.algaebase.org (accessed on 5 June 2021).

37. Sládeèek, V. Diatoms as indicators of organic Pollution. Acta Hydrochim. Et Hydrobiol. 1986, 14, 555-566. [CrossRef]

38. Getsen, M.V.; Stenina, A.S. Algae (Systematic List): In Flora and Fauna of Water Bodies in the European North; Nauka: Leningrad, Russia, 1978; pp. 109-150. (In Russian)

39. Van Dam, H.; Mertens, A.; Sinkeldam, J. A coded checklist and ecological indicator values of freshwater diatoms from the Netherlands. Netherland J. Aquat. Ecol. 1994, 1, 117-133.

40. Barinova, S.S.; Medvedeva, L.A. Atlas of Algae as Saprobic Indicators (Russian Far East); Dal'nauka Press: Vladivostok, Russia, 1996; p. 364. (In Russian)

41. Barinova, S.S.; Medvedeva, L.A.; Anisimova, O.V. Algae as Indicators of Environmental Quality; Institute Nature Conservation Press: Moscow, Russia, 2000; p. 150. (In Russian) 
42. Barinova, S.S.; Medvedeva, L.A.; Anisimova, O.V. Biodiversity of Environmental Indicator Algae; Pilies Studio: Tel Aviv, Israel, 2006; p. 498. (In Russian)

43. Loseva, E.I.; Stenina, A.S.; Marchenko-Vagapova, T.I. Cadastre of the Fossil and Recent Diatoms from Northeastern Europe; Geoprint: Syktyvkar, Russia, 2004; p. 160. (In Russian)

44. Stenina, A.S.; Sterlyagova, I.N. Bacillariophyta in epilithon of the Shchugor River (Urals, Komi Republic). Bot. Zhurnal 2017, 102, 1107-1122. [CrossRef]

45. Stenina, A.S. Annotated list of Bacillariophyta of the Shchugor River (Urals, Komi Republic). Bot. Zhurnal 2019, 104, 41-57. [CrossRef]

46. Gavshin, V.M.; Arkhipov, S.A.; Bobrov, V.A.; Melgunov, M.S.; Makarova, I.V.; Sukhorukov, F.V.; Stepin, A.S.; Khlystov, O.M.; Kireev, A.D.; Budashkina, V.V. Distribution of natural radioactive elements in Holocene-Pleistocene deep-water sediments of Lake Baikal and paleoclimate reconstruction. Geol. Geophys. 1998, 39, 1045-1058.

47. Korotkiy, A.M.; Grebennikova, T.A.; Pushkar, B.C.; Razzhigaeva, N.G.; Volkov, V.G.; Ganzei, L.A.; Mokhova, L.M.; Bazarova, V.B.; Makarova, T.R. Climatic Changes in the South of the Far East in the Late Cenozoic (Miocene-Pleistocene); Publishing House of the Far Eastern State University: Vladivostok, Russia, 1996; p. 56. (In Russian)

48. Lavrushin, Y.A.; Alekseev, M.N. Late Cenozoic paleoclimatic events in the Arctic ocean. Stratigraphy. Geol. Correl. 1993, 1, 48-58.

49. Pushkar, V.S.; Cherepanova, M.V. Diatoms of the Pliocene and Anthropogen of the Northern Pacific (Stratigraphy and Paleoecology); Dal'nauka: Vladivostok, Russia, 2001; p. 226. (In Russian)

50. Baskakova, L.A. Eventfulness and stages of Geological development in the Cenozoic of the Mainland part of the East of Russia. Pac. Geol. 2006, 25, 3-18.

51. Zabelina, M.M.; Kiselev, I.A.; Proshkina-Lavrenko, A.I.; Sheshukova, V.S. Diatoms Keys to Freshwater Algae of the USSR; Soviet Science: Moscow, Russia, 1951; p. 619. (In Russian)

52. Popovskaya, G.I.; Genkal, S.I.; Likhoshway, Y.V. Diatoms of the Plankton of Lake Baikal: Atlas and Key; Nauka: Novosibirsk, Russia, 2016; p. 180. (In Russian)

53. Khursevich, G.K.; Karabanov, E.B.; Prokopenko, A.A.; Williams, D.F.; Kuz'min, M.I.; Fedenya, S.A.; Gvozdkov, A.N.; Kerber, E.V. Detailed diatom biostratigraphy of Baikal sediments during the brunhes chron and climatic factors of species formation. Russ. Geol. Geophys. 2001, 42, 108-129.

54. Babanazarova, O.V.; Likhoshway, Y.V.; Sherbakov, D.Y. On the morphological variability of Aulacoseira baicalensis and Aulacoseira islandica (Bacillariophyta) of Lake Baikal, Russia. Phycologia 1996, 35, 113-123. [CrossRef]

55. Endrikhinsky, A.S.; Cheremisinova, E.A. On the finding of Miocene deposits on the Vitim Plateau. Dokl. Acad. Sci. USSR 1970, 1, 885-888.

56. Chernyaeva, G.P.; Lyamina, N.A.; Rasskazov, S.V. Biostratigraphy and conditions of accumulation of the Middle and Upper Miocene volcanogenic-sedimentary strata of the Dzhilinda depression in western Transbaikalia. Geol. Geophys. 2007, 48, 460-471. 